MATHEMATICS OF COMPUTATION

Volume 75, Number 256, October 2006, Pages 1931-1970

S 0025-5718(06)01861-8

Article electronically published on May 31, 2006

\title{
ITERATED FUNCTION SYSTEMS, RUELLE OPERATORS, AND INVARIANT PROJECTIVE MEASURES
}

\author{
DORIN ERVIN DUTKAY AND PALLE E. T. JORGENSEN
}

\begin{abstract}
We introduce a Fourier-based harmonic analysis for a class of discrete dynamical systems which arise from Iterated Function Systems. Our starting point is the following pair of special features of these systems. (1) We assume that a measurable space $X$ comes with a finite-to-one endomorphism $r: X \rightarrow X$ which is onto but not one-to-one. (2) In the case of affine Iterated Function Systems (IFSs) in $\mathbb{R}^{d}$, this harmonic analysis arises naturally as a spectral duality defined from a given pair of finite subsets $B, L$ in $\mathbb{R}^{d}$ of the same cardinality which generate complex Hadamard matrices.

Our harmonic analysis for these iterated function systems (IFS) $(X, \mu)$ is based on a Markov process on certain paths. The probabilities are determined by a weight function $W$ on $X$. From $W$ we define a transition operator $R_{W}$ acting on functions on $X$, and a corresponding class $H$ of continuous $R_{W^{-}}$ harmonic functions. The properties of the functions in $H$ are analyzed, and they determine the spectral theory of $L^{2}(\mu)$. For affine IFSs we establish orthogonal bases in $L^{2}(\mu)$. These bases are generated by paths with infinite repetition of finite words. We use this in the last section to analyze tiles in $\mathbb{R}^{d}$.
\end{abstract}

\section{INTRODUCTION}

One of the reasons wavelets have found so many uses and applications is that they are especially attractive from the computational point of view. Traditionally, scale/translation wavelet bases are used in function spaces on the real line, or on Euclidean space $\mathbb{R}^{d}$. Since we have Lebesgue measure, the Hilbert space $L^{2}\left(\mathbb{R}^{d}\right)$ offers the natural setting for harmonic analysis with wavelet bases. These bases can be made orthonormal in $L^{2}\left(\mathbb{R}^{d}\right)$, and they involve only a fixed notion of scaling, for example by a given expansive $d$-by- $d$ matrix $A$ over $\mathbb{Z}$, and translation by the integer lattice $\mathbb{Z}^{d}$. But this presupposes an analysis that is localized in a chosen resolution subspace, say $V_{0}$ in $L^{2}\left(\mathbb{R}^{d}\right)$. That this is possible is one of the successes of wavelet computations. Indeed, it is a nontrivial fact that a rich variety of such subspaces $V_{0}$ exist, and further that they may be generated by one, or a finite set of functions $\varphi$ in $L^{2}\left(\mathbb{R}^{d}\right)$ which satisfy a certain scaling equation [Dau92].

Received by the editor January 5, 2005 and, in revised form, June 16, 2005.

2000 Mathematics Subject Classification. Primary 28A80, 31C20, 37F20, 39B12, 41A63, 42C40, 47D07, 60G42, 60J45.

Key words and phrases. Measures, projective limits, transfer operator, martingale, fixed-point, wavelet, multiresolution, fractal, Hausdorff dimension, Perron-Frobenius, Julia set, subshift, orthogonal functions, Fourier series, Hadamard matrix, tiling, lattice, harmonic function.

This research was supported in part by the National Science Foundation DMS-0139473 (FRG). 
The determination of this equation might only involve a finite set of numbers (four-tap, six-tap, etc.), and it is of central importance for computation. The solutions to a scaling equation are called scaling functions, and are usually denoted $\varphi$. Specifically, the scaling equation relates in a well-known way the $A$-scaling of the function(s) $\varphi$ to their $\mathbb{Z}^{d}$-translates.

The fact that there are solutions in $L^{2}\left(\mathbb{R}^{d}\right)$ is not at all obvious; see Dau92. In application to images, the subspace $V_{0}$ may represent a certain resolution, and hence there is a choice involved, but we know by standard theory (see, e.g., Dau92]) that under appropriate conditions such choices are possible. As a result there are extremely useful, and computationally efficient, wavelet bases in $L^{2}\left(\mathbb{R}^{d}\right)$. A resolution subspace $V_{0}$ within $L^{2}\left(\mathbb{R}^{d}\right)$ can be chosen to be arbitrarily fine: finer resolutions correspond to larger subspaces.

As noted for example in [BrJo02, a variant of the scaling equation is also used in computer graphics: there data is successively subdivided, and the refined level of data is related to the previous level by prescribed masking coefficients. The latter coefficients in turn induce generating functions which are direct analogues of wavelet filters; see the discussions in Section 3 and at the end of Section 5 .

One reason for the computational efficiency of wavelets lies in the fact that wavelet coefficients in wavelet expansions for functions in $V_{0}$ may be computed using matrix iteration, rather than by a direct computation of inner products: the latter would involve integration over $\mathbb{R}^{d}$, and hence be computationally inefficient, if feasible at all. The deeper reason why we can compute wavelet coefficients using matrix iteration is an important connection to the subband filtering method from signal/image processing involving digital filters, down-sampling, and up-sampling. In this setting filters may be realized as functions $m_{0}$ on a $d$-torus, e.g., quadrature mirror filters; see details below.

As emphasized for example in [Jo05a, because of down-sampling, the matrix iteration involved in the computation of wavelet coefficients involves so-called slanted Toeplitz matrices $F$ from signal processing. The slanted matrices $F$ are immediately avaliable; they simply record the numbers (masking coefficients) from the $\varphi$-scaling equation. These matrices further have the computationally attractive property that the iterated powers $F^{k}$ become sucessively more sparse as $k$ increases, i.e., the matrix representation of $F^{k}$ has mostly zeros, and the nonzero terms have an especially attractive geometric configuration. In fact subband signal processing yields a finite family, $F, G$, etc., of such slanted matrices, and the wavelet coefficients at scaling level $k$ of a numerical signal $s$ from $V_{0}$ are then simply the coordinates of $G F^{k} s$. By this we mean that a signal in $V_{0}$ is represented by a vector $s$ via a fixed choice of scaling function; see [Dau92, BrJo02]. Then the matrix product $G F^{k}$ is applied to $s$, and the matrices $G F^{k}$ get more slanted as $k$ increases.

Our paper begins with the observation that the computational feature of this engineering device can be said to begin with an endomorphism $r_{A}$ of the $d$-torus $\mathbb{T}^{d}=\mathbb{R}^{d} / \mathbb{Z}^{d}$, an endomorphism which results from simply passing matrix multiplication by $A$ on $\mathbb{R}^{d}$ to the quotient by $\mathbb{Z}^{d}$. It is then immediate that the inverse images $r_{A}^{-1}(x)$ are finite for all $x$ in $\mathbb{T}^{d}$, in fact $\# r_{A}^{-1}(x)=|\operatorname{det} A|$. From this we recover the scaling identity, and we note that the wavelet scaling equation is a special case of a more general identity known in computational fractal theory and in symbolic dynamics. We show that wavelet algorithms and harmonic analysis naturally generalize to affine iterated function systems. Moreover, in this general context, 
we are able to build the ambient Hilbert spaces for a variety of dynamical systems which arise from the iterated dynamics of endomorphisms of compact spaces.

As a consequence, the fact that the ambient Hilbert space in the traditional wavelet setting is the more familiar $L^{2}\left(\mathbb{R}^{d}\right)$ is merely an artifact of the choice of filters $m_{0}$. As we further show, by enlarging the class of admissible filters, there are a variety of other ambient Hilbert spaces possible with corresponding wavelet expansions: the most notable are those which arise from iterated function systems (IFS) of fractal type, for example for the middle-third Cantor set, and scaling by 3; see Example 5.16 .

More generally (see Section 7), there are a variety of other natural dynamical settings (affine IFSs) that invite the same computational approach (Sections 869).

The two most striking examples which admit such a harmonic analysis are perhaps complex dynamics and subshifts. Both will be worked out in detail inside the paper. In the first case, consider a given rational function $r(z)$ of one complex variable. We then get an endomorphism $r$ acting on an associated Julia set $X$ in the complex plane $\mathbb{C}$ as follows. This endomorphism $r: X \rightarrow X$ results by restriction to $X$ Bea91. (Details: Recall that $X$ is by definition the complement of the points in $\mathbb{C}$ where the sequence of iterations $r^{n}$ is a normal family. Specifically, the Fatou set $F$ of $r(z)$ is the largest open set in $\mathbb{C}$ where $r^{n}$ is a normal sequence of functions, and we let $X$ be the complement of $F$. Here $r^{n}$ denotes the $n$th iteration of the rational function $r(z)$.) The induced endomorphism $r$ of $X$ is then simply the restriction to $X$ of $r(z)$. If $r$ then denotes the resulting endomorphism, $r: X \rightarrow X$, it is known DuJo04a] that $\# r^{-1}(x)=$ degree of $r$, for every $x$ in $X$ (except for a finite set of singular points).

In the second case, for a particular one-sided subshift, we may take $X$ as the corresponding state space, and again we have a naturally induced finite-to-one endomorphism of $X$ of geometric and computational significance.

But in the general framework, there is not a natural candidate for the ambient Hilbert space. That is good in one sense, as it means that the subband filters $m_{0}$ which are feasible will constitute a richer family of functions on $X$.

In all cases, the analysis is governed by a random-walk model with successive iterations where probabilities are assigned on the finite sets $\# r^{-1}(x)$ and are given by the function $W:=\left|m_{0}\right|^{2}$. This leads to a transfer operator $R_{W}$ (see (2.1) below) which has features in common with the classical operator considered first by Perron and Frobenius for positive matrices. In particular it has a PerronFrobenius eigenvalue and positive Perron-Frobenius eigenvectors, one on the right, a function, and one on the left, a measure; see Rue89. As we show in Section 5. this Perron-Frobenius measure, also sometimes called the Ruelle measure, is an essential ingredient for our construction of an ambient Hilbert space. All of this, we show, applies to a variety of examples, and as we show, has the more traditional wavelet setup as a special case, in fact the special case when the Ruelle measure on $\mathbb{T}^{d}$ is the Dirac mass corresponding to the point 0 in $\mathbb{T}^{d}$ (additive notation) representing zero frequency in the signal processing setup.

There are two more ingredients entering into our construction of the ambient Hilbert space: a path space measure governed by the $W$-probablities, and certain finite cycles for the endomorphism $r$; see Sections 2 and 4 . For each $x$ in $X$, we consider paths by infinite iterated tracing back with $r^{-1}$ and recursively assigning probabilities with $W$. Hence we get a measure $P_{x}$ on a space of paths for each $x$. 
These measures are in turn integrated in $x$ using the Ruelle measure on $X$. The resulting measure will now define the inner product in the ambient Hilbert space.

Our present harmonic analysis for these systems is governed by a certain class of geometric cycles for $r$, i.e., cycles under iteration by $r$. We need cycles where the function $W$ attains its maximum, and we call them $W$-cycles. They are essential, and our paper begins with a discussion of $W$-cycles for particular examples, including their geometry, and a discussion of their significance for the computation in an orthogonal harmonic analysis; see especially Theorem 8.4 and Remark 8.5. Theorem 8.4 is one of our main results. It gives a necessary and sufficient condition for a certain class of affine fractals in $\mathbb{R}^{d}$ to have an orthonormal Fourier basis, and it even gives a recipe for what these orthonormal bases look like. We believe that this theorem throws new light on a rather fundamental question: which fractals admit complete sets of Fourier frequencies? Our result further extends earlier work by a number of authors; in particular, it clarifies the scale-4 Cantor set (Remark 8.5) on the line, considered earlier by the second author and S. Pedersen [JoPe98, and also by R. Strichartz [Str00, Str05], and I. Laba and Y. Wang [LaWa02].

\section{Probabilities on Path SPACE}

This paper is motivated by our desire to apply wavelet methods to some nonlinear problems in symbolic and complex dynamics. Recent research by many authors (see, e.g., AST04 and ALTW04) on iterated function systems (IFS) with affine scaling have suggested that the scope of the multiresolution method is wider than the more traditional wavelet context, where it originated in the 1980s; see [Dau92].

In this paper we concentrate on a class of iterate function systems (IFS) considered earlier in [Hut81, JoPe96], JoPe98, Jo05b], Str00, and LaWa02.

These are special cases of discrete dynamical systems which arise from a class of Iterated Function Systems; see [YHK97. Our starting point is two features of these systems which we proceed to outline.

(1) In part of our analysis, we suppose that a measurable space $X$ comes with a fixed finite-to-one endomorphism $r: X \rightarrow X$, which is assumed onto but not oneto-one. (Such systems arise for example as Julia sets in complex dynamics where $r$ may be a rational mapping in the Riemann sphere and $X$ the corresponding Julia set, but also as affine iterated function systems from geometric measure theory.)

In addition, we suppose $X$ comes with a weighting function $W$ which assigns probabilities to a certain branching tree $\left(\tau_{\omega}\right)$ defined from the iterated inverse images under the map $r$. This allows us to define a Ruelle operator $R_{W}$ acting on functions on $X$,

$$
R_{W} f(x)=\sum_{y \in r^{-1}(x)} W(y) f(y), \quad x \in X
$$

or more generally

$$
R_{W} f(x)=\sum_{\omega} W\left(\tau_{\omega} x\right) f\left(\tau_{\omega} x\right),
$$

and an associated class of $R_{W}$-harmonic functions on $X$ (Section 4.1).

Assembling the index-system for the branching mappings $\tau_{i}$, we get an infinite Cartesian product $\Omega$. Points in $\Omega$ will be denoted $\omega=\left(\omega_{1}, \omega_{2}, \ldots\right)$. (The simplest case is when $\# r^{-1}(x)$ is a finite constant $N$ for all but a finite set of points in $X$. 
In that case

$$
\Omega:=\prod_{1}^{\infty} \mathbb{Z}_{N}
$$

where $\mathbb{Z}_{N}$ is the finite cyclic qroup of order $N$.)

Our interest lies in a harmonic analysis on $(X, r)$ which begins with a PerronFrobenius problem for $R_{W}$. Much of the earlier work in this context (see, e.g., Ba00, Rue89, and MaUr04]) is restricted to the case when $W$ is strictly positive, but here we focus on when $W$ assumes the value zero on a finite subset of $X$. We then show that generically the Perron-Frobenius measures (typically nonunique) have a certain dichotomy. When the $(X, r)$ has iterated backward orbits which are dense in $X$, then the ergodic Perron-Frobenius measures either have full support, or else their support is a union of cycles defined from $W$ (see Definition 2.2 and Section (4).

(2) In the case of affine Iterated Function Systems in $\mathbb{R}^{d}$ (Sections 179), this structure arises naturally as a spectral duality defined from a given pair of finite subsets $B, L$ in $\mathbb{R}^{d}$ of the same cardinality which generate complex Hadamard matrices (Section 7). When the system $(B, L)$ is given, we first outline the corresponding construction of $X, r, W$, and a family of probability measures $P_{x}$. We then show how the analysis from (1) applies to this setup (which also includes a number of multiresolution constructions of wavelet bases). This in turn is based on a certain family of path-space measures, i.e., measures $P_{x}$, defined on certain projective limit spaces $X_{\infty}(r)$ of paths starting at points in $X$, and depending on $W$. The question of when there are scaling functions for these systems depends on certain limit sets of paths with repetition in $X_{\infty}(r)$ having full measure with respect to each $P_{x}$.

Our construction suggests a new harmonic analysis, and wavelet basis construction, for concrete Cantor sets in one and higher dimensions.

The study of the $(B, L)$-pairs which generate complex Hadamard matrices (see Definition 3.4) is of relatively recent vintage. These pairs arose first in connection with a spectral problem of Fuglede [Fu74, and their use was first put to the test in Jo82 and [JoPe92.

We include a brief discussion of it below.

In [JoPe98, Pedersen and the second named author found that there are two nontrivial kinds of affine IFSs, those that have the orthonormal basis (ONB) property with respect to a certain Fourier basis (such as the quarter-Cantor set, scaling constant $=4$, \#subdivisions = 2) and those that do not (such as the middle-third Cantor set, scaling constant $=3$, \#subdivisions $=2$ ).

Definition 2.1. Let $X \subset \mathbb{R}^{d}$ be a compact subset, let $\mu$ be a Borel probability measure on $X$, i.e., $\mu(X)=1$, and let $L^{2}(X, \mu)$ be the corresponding Hilbert space. We say that $(X, \mu)$ has an ONB of Fourier frequencies if there is a subset $\Lambda \subset \mathbb{R}^{d}$ such that the functions $e_{\lambda}(x)=e^{i 2 \pi \lambda \cdot x}, \lambda \in \Lambda$, form an orthonormal basis for $L^{2}(X, \mu)$, referring to the restriction of the functions $e_{\lambda}$ to $X$.

Definition 2.2. Let $(X, r, W)$ be as described above, with $R_{W} 1=1$. Suppose there are $x \in X$ and $n \in \mathbb{N}$ such that $r^{n}(x)=x$. Then we say that the set $C_{x}:=\left\{x, r(x), \ldots, r^{n-1}(x)\right\}$ is an $n$-cycle. (When referring to an $n$-cycle $C$, it is understood that $n$ is the smallest period of $C$.) We say that $C_{x}$ is a $W$-cycle if it is an $n$-cycle for some $n$, and $W(y)=1$ for all $y \in C_{x}$. 
Because of the fractal nature of the examples, in fact it seems rather surprising that any affine IFSs have the ONB/Fourier property at all. The paper [JoPe98. started all of this, i.e., Fourier bases on affine fractals, and it was found that these classes of systems may be based on our special $(B, L)$-Ruelle operator, i.e., they may be defined from $(B, L)$-Hadamard pairs [JoPe92] and an associated Ruelle operator Rue89.

There was an initial attempt to circumvent the Ruelle operator (e.g., Str98] and Str00]) and an alternative condition for when we have a Fourier ONB emerged, based on an idea of Albert Cohen (see Dau92]). The author of [Str00 and [Str05] names these ONBs "mock Fourier series".

Subsequently there was a follow-up paper by I. Laba and Y. Wang LaWa02 which returned the focus to the Ruelle operator from JoPe98.

In the present paper, we continue the study of the $(B, L)$-Hadamard pairs (see Definition (3.4) in a more general context than for the special affine IFSs that have the ONB property. That is because the ONB property entails an extra integrality condition which we are not imposing here. As a result we get the Ruelle operator setting to work for a wide class of $(B, L)$-Hadamard pairs. This class includes everything from the earlier papers (in particular, it includes the middle-third Cantor set example, i.e., the one that does not have any Fourier ONB!).

Nonetheless the setting of Theorem 1.3 in LaWa02 fits right into our present context.

In Sections 7 9, we consider the affine IFSs, and we place a certain Lipschitz condition on the weight function $W$.

We prove that if $W$ is assumed Lipschitz, the inverse branches of the endomorphism $r: X \rightarrow X$ are contractive, and there exist some $W$-cycles, then the dimension of the eigenspace $R_{W} h=h$ with $h$ continuous is equal to the number of $W$-cycles. In the $r(z)=z^{N}$ case, this is similar to a result in Conze-Raugi CoRa90. Conze and Raugi state in CoRa90 that this philosophy might work under some more general assumptions, perhaps for the case of branches from a contractive IFS. Here we show that we do have it under a more general hypothesis, which includes the subshifts and the Julia sets.

When $W$ is specified (see details Section 6), we study the $W$-cycles. For each $W$-cycle $C$, we get an $R_{W}$-harmonic function $h_{C}$, i.e., $R_{W} h_{C}=h_{C}$, and we are able to conclude, under a certain technical condition (TZ), that the space of all $R_{W}$-harmonic functions is spanned by $h_{C}$ functions. In fact every positive (i.e., nonnegative) harmonic function $h$ such that $h \leq 1$ is a convex combination of $h_{C}$ functions. In Section 9, we introduce a class of planar systems $(B, L, R)$, i.e., $d=2$, where the condition (TZ) is not satisfied, and where there are $R_{W}$-harmonic functions which are continuous, but which are not spanned by the special functions $h_{C}$, indexed by the $W$-cycles.

With this theorem, we show the harmonic functions $h$, i.e., $R_{W} h=h$, to be of the form $h(x)=P_{x}(\mathbb{N})$, where, for each $W$-cycle, a copy of $\mathbb{N}$ is naturally embedded in $\Omega$. For each cycle, there is a harmonic function, and thus the sum of them is the constant function 1. Then, in the case of just one cycle, we recover the result of [LaWa02, Theorem 1.3]. We will also get as a special case the wellknown orthogonality condition for the scaling function of a multiresolution wavelet (see [Dau92, Chapter 6]). We know that the case of multiple cycles gives the superwavelets (see [BDP05]), but the case of the affine IFS, with $W$ coming from 
the Hadamard matrix, yields interesting and unexpected spectra for associated spectral measures (Sections 8 91). We further study the zeroes of the functions $x \rightarrow P_{x}(\mathbb{N})$, and

$$
x \rightarrow P_{x}(\text { cycle.cycle.cycle } \cdots)
$$

for various cycles, and relate them to the spectrum. By the expression in (2.3), we mean an infinite repetition of a finite word.

Remark 2.3. For a given system $(X, W)$ we stress the distinction between the general $n$-cycles and the $W$-cycles; see Definition 2.2. While the union of the $n$ cycles is infinite, the IFSs we study in this paper typically have only finite sets of $W$-cycles; see Section 6 and the examples in Section 9 below. When $X$ is given, intuitively, the union over $n$ of all the $n$-cycles is a geometric analogue of the set of rational fractions for the usual positional number system, and it is typically dense in $X$. But when $W$ is also given as outlined, and continuous, then we show that the $W$-cycles determine the harmonic analysis of the transfer operator $R_{W}$, acting on the space of continuous functions on $X$. This result generalizes two theorems from the theory of wavelets; see [Dau92, Theorems 6.3.5, and 6.3.6].

While our focus here is the use of the transfer operator in the study of wavelets and IFSs, it has a variety of other but related applications, see, e.g., Ba00, NuLu99, Che99, MaUr04, Wal75], LMW96, LWC95, Law91.

In the encoding of (2.3), copies of the natural numbers $\mathbb{N}$ are represented as subsets in $\Omega$ (see (2.2) ) consisting of all finite words, followed by an infinite string of zeros, or more generally by an infinite repetition of some finite cycle; see Proposition 8.1 .

The idea of identifying classes of $R_{W}$-harmonic functions for IFSs with the use of path space measures and cocycles, along the lines of (2.3), was first put forth in a very special case by R. Gundy in the wavelet context. This was done in three recent and original papers by R. Gundy Gu99, Gu00, GuKa00, and our present results are much inspired by Richard Gundy's work. Gundy's aim was to generalize and to offer the correct framework for the classical orthogonality conditions for translation/scale wavelets, first suggested in papers by A. Cohen and W. Lawton; see [Dau92, Chapter 5] for details. We are pleased to acknowledge helpful discussions with Richard Gundy on the subject of our present research.

\section{Definitions AND BaCKGRound}

For the applications we have in mind, the following setting is appropriate. The space $X$ arises as a closed subspace in a complete metric space $(Y, d)$. For each $x \in X$, there is a finite and locally defined system of measurable mappings $\left(\tau_{i}\right)$ such that $r \circ \tau_{i}=$ id holds in a neighborhood of $x$. Our results in the second half of the paper apply to the general case of IFSs, i.e., even when such an endomorphism $r$ is not assumed. Note that if $r$ exists, then the sets $\tau_{i}(X)$ are mutually disjoint.

This construction is motivated by DuJo05. To see this, let $r$ be an endomorphism in a compact metric space $X$ (for example the Julia set Bea91 of a given rational map $w=r(z)$ ), and suppose $r$ is onto $X$ and finite-to-one. Form a projective space $P=P(X, r)$ such that $r$ induces an automorphism $a=a(r)$ of $P(X, r)$. Let $W$ be a Borel function on $X$ (naturally extended to a function on $P)$. Generalizing the more traditional approach to scaling functions, we found in DuJo05] a complete classification of measures on $P(X, r)$ which are quasi-invariant 
under $a(r)$ and have Radon-Nikodym derivative equal to $W$. Our analysis of the quasi-invariant measures is based on certain Hilbert spaces of martingales, and on a transfer operator (equation (2.1)) studied first by David Ruelle Rue89.

For the application to iterated function systems (IFS), the following condition is satisfied. For every $\omega=\left(\omega_{1}, \omega_{2}, \ldots\right) \in \Omega$, the intersection

$$
\bigcap_{n=1}^{\infty} \tau_{\omega_{1}} \cdots \tau_{\omega_{n}}(Y)
$$

is a singleton $x=\pi(\omega)$, and $x$ is in $X$ (see Section 4.2 for details).

Definition 3.1. The shift on $\Omega,\left(\omega_{1}, \omega_{2}, \ldots\right) \mapsto\left(\omega_{2}, \omega_{3}, \ldots\right)$ will be denoted $r_{\Omega}$, and it is clear that

$$
\# r_{\Omega}^{-1}(\omega)=N
$$

for all $\omega \in \Omega$.

In the general context of IFSs $\left(X,\left(\tau_{i}\right)_{i=1}^{N}\right)$, as in (3.1), we may introduce the backward orbit and cycles as follows. Set

$$
C^{-n}(x):=\pi\left(r_{\Omega}^{-n}\left(\pi^{-1}(x)\right)\right), \quad x \in X .
$$

If $x \in X$ and $p \in \mathbb{N}$, we say that $C(x)$ is a cycle of length $p$ for $\left(X,\left(\tau_{i}\right)_{i=1}^{N}\right)$ if there is a cycle of length $p, C_{\Omega}(\omega)$ in $\Omega$ for some $\omega \in \pi^{-1}(x)$ such that

$$
C(x)=\pi\left(C_{\Omega}(\omega)\right) .
$$

Remark 3.2. We must assume that intersections in (3.1) collapse to a singleton. Start with a given infinite word, $\omega=\left(\omega_{1}, \omega_{2}, \ldots\right)$, and define composite maps from an IFS consisting of contractive maps in a suitable space $Y$. The finitely composite maps are applied to $Y$, and they correspond to finite words indexed from 1 to $n$. Then there is an intersection over $n$, as the finite words successively fill out more of the fixed infinite word $\omega$. That will be consistent with the usual formulas for the positional convention in our representation of real numbers, in some fixed basis, i.e., an finite alphabet $A$, say $A=\{0,1\}$, or some other finite $A$. We will even allow the size of $A$ to vary locally. This representation of IFSs is discussed in more detail in, for example [YHK97, page 30] and [AtNe04.

Definition 3.3. The condition that the intersection in (3.1) is a singleton will be assumed throughout, and the corresponding mapping $\pi: \Omega \rightarrow X$ will be assumed to be onto. It is called the symbol mapping of the system $(X, r)$.

We shall further assume that the definition

$$
\omega \sim \omega^{\prime} \Leftrightarrow \pi(\omega)=\pi\left(\omega^{\prime}\right)
$$

yields an equivalence relation on the symbol space $\Omega$. As a result $\Omega / \sim$ will serve as a model for $X$.

Let $\mathbb{S}$ be the Riemann sphere (i.e., the one-point compactification of $\mathbb{C}$ ), and let $r$ be a fixed rational mapping. The $n$-fold iteration of $r$ will be denoted $r^{n}$. Let $\mathcal{U}$ be the largest open set in $\mathbb{S}$ for which $\left.r^{n}\right|_{\mathcal{U}}$ is a normal family. Then the complement $X:=\mathbb{S} \backslash \mathcal{U}$ is the Julia set. It is known Bro65 that if $N$ is the degree of $r$, then the above condition is satisfied for the pair $(X, r)$, i.e., referring to the restriction to $X$ of the fixed rational mapping $r$. 
For a general system $(X, r)$ as described, we define the backward orbit $O^{-}(x)$ of a point $x$ in $X$ as

$$
O^{-}(x):=\bigcup_{n=1}^{\infty} r^{-n}(x)
$$

where $r^{-n}(x)=\left\{y \in X \mid r^{n}(y)=x\right\}$.

For concrete iteration systems $(X, r)$ of quasi-regular mappings, conditions are known for when there are backward orbits $O^{-}(x)$ which are dense in $X$; see HMM04.

Definition 3.4. Following JoPe98, we consider two subsets, $B, L$, in $\mathbb{R}^{d}$ for some $d \geq 1$. We say that the sets form a Hadamard pair if $\# B=\# L=N$, and if the matrix

$$
U:=\frac{1}{\sqrt{N}}\left(e^{2 \pi i b \cdot l}\right)_{b \in B, l \in L}
$$

is unitary, i.e., $U^{*} U=I=$ (the identity matrix).

We have the occasion to use the two finite sets $B$ and $L$ from a Hadamard pair $(B, L)$ in different roles. One set serves as translation vectors of one IFS, and the other in a role of specifying $W$-frequencies for the weight function $W$ of $R_{W}$. So on the one hand we have a pair with $\left\{\tau_{b} \mid b \in B\right\}$ as an IFS and $W_{L}$ as a corresponding weight function, and on the other hand, a different IFS $\left\{\tau_{l} \mid l \in L\right\}$ with a corresponding $W_{B}$.

Example 3.5. The Fourier transform of the finite cyclic group $\mathbb{Z}_{N}$ of order $N$ has the form

$$
\frac{1}{\sqrt{N}}\left(\xi_{N}^{k l}\right)_{k, l=0}^{N-1}, \quad \text { where } \xi_{N}=e^{i \frac{2 \pi}{N}} .
$$

But there are other complex Hadamard matrices. For example if $U$ is an $N \times N$ and $V$ is an $M \times M$ complex Hadamard matrix, then $U \otimes V$ is a complex $(N M) \times(N M)$ Hadamard matrix. Using this rule twice we get the following family of Hadamard matrices:

$$
\left(\begin{array}{cccc}
1 & 1 & 1 & 1 \\
1 & 1 & -1 & -1 \\
1 & -1 & u & -u \\
1 & -1 & -u & u
\end{array}\right), \quad u \in \mathbb{T} .
$$

To each Hadamard matrix, there is a rich family of IFSs of the form $(B, L)$ as in (3.2); see [JoPe96] for details.

Complex Hadamard matrices have a number of uses in combinatorics SeYa92. and in physics Wer93, RW96.

The correspondence principle $B \leftrightarrow L$ is pretty symmetric except that the formula we use for $\left\{\tau_{b} \mid b \in B\right\}$ is a little different from that for $\left\{\tau_{l} \mid l \in L\right\}$. The reason for this asymmetry is outlined in [JoPe98, where we also had the occasion to use both systems. Here and in [JoPe98, the matrix $R$ transforms the two sets $B$ and $L$ in a certain way (see (8.1)-(8.2) ), and that is essential in our iteration schemes. Our present setup is more general.

The connection from Hadamard pairs to IFS is outlined in JoPe98 and recalled below. 
Definition 3.6. Let $d \in \mathbb{N}$ be given. We say that $(B, L, R)$ is a system in Hadamard duality if

- $B$ and $L$ are subsets of $\mathbb{R}^{d}$ such that $\# B=\# L=: N$;

- $R$ is some fixed $d \times d$ matrix over $\mathbb{R}$ with all eigenvalues $\lambda$ satisfying $|\lambda|>1$;

- the sets $\left(R^{-1} B, L\right)$ form a Hadamard pair (with an $N \times N$ Hadamard matrix).

Then we let

- $\tau_{b}(x):=R^{-1}(b+x), x \in \mathbb{R}^{d}$;

- $\tau_{l}(x):=S^{-1}(l+x), x \in \mathbb{R}^{d} ; S=R^{t}$ (the transpose matrix);

- $X_{B}$ will then be the unique compact subset such that

$$
X_{B}=\bigcup_{b \in B} \tau_{b}\left(X_{B}\right)
$$

or equivalently

$$
R X_{B}=X_{B}+B .
$$

(Recall that the symbol space $\Omega$ for $X$ in this case is $\Omega=\prod_{0}^{\infty} \mathbb{Z}_{N}$, or since $\# B=N, \Omega=\prod_{0}^{\infty} B$.)

Setting

$$
m_{B}(x)=\frac{1}{\sqrt{N}} \sum_{b \in B} e^{2 \pi i b \cdot x}
$$

and $W_{B}(x):=\left|m_{B}(x)\right|^{2} / N$, it follows that

$$
\sum_{l \in L} W_{B}\left(\tau_{l} x\right)=1, \quad x \in \mathbb{R}^{d} .
$$

Remark 3.7. Consider this setup in one dimension. The question of when a pair of two-element sets will generate a complex 2-by-2 Hadamard matrix as in (3.2) may be understood as follows: Set

$$
U=\frac{1}{\sqrt{2}}\left(\begin{array}{cc}
1 & 1 \\
1 & -1
\end{array}\right) .
$$

Without loss of generality, we may take $B=\{0, b\}, L=\{0, l\}$; then $N=2=$ $\# B=\# L$. Also, the case of a scale with the number 4 , i.e., $R=4$, is of special significance.

To get the Hadamard property for the system $(B, L, R)$ we must have $4^{-1} a b=\frac{1}{2}$ $\bmod 1$, so we may take $b=2$ and $l=1$. Then we get an orthonormal basis (ONB) of Fourier frequencies in the associated iterated function system (IFS): $\{x / 4,(x+2) / 4=x / 4+1 / 2\}$ and induced Hilbert space $L^{2}(\mu)$, corresponding to Hausdorff measure $\mu$ of Hausdorff dimension 1/2. Recall that the Hausdorff measure is in fact restricted to the fractal $X$, and $\mu(X)=1$; see JoPe98 and Hut81. We then get an ONB in $L^{2}(X, \mu)$ built from $L$ and the scale number 4 as follows. The ONB is of the form $e_{\lambda}:=\exp (i 2 \pi \lambda x)$, where $\lambda$ ranges over $\Lambda:=\{0,1,4,5,16,17,20,21,24,25, \ldots\}$; see Section 8 , Remark 8.5 , for a full analysis.

If instead we take $B=\{0, b\}, L=\{0, l\}$, but we scale with 3 , or with any odd integer, then by [JoPe98, we cannot have more than two orthogonal Fourier frequencies. So certainly there is not an ONB in the corresponding $L^{2}(\mu), \mu=$ Hausdorff measure of dimension $\log _{3}(2)$, consisting of Fourier frequencies $e_{\lambda}$ for any choice of $\lambda$. 


\section{SETUP}

There are two situations that we have in mind:

(1) The first one involves an iterated function system $\left(\tau_{i}\right)_{i=1}^{N}$ on some compact metric space.

(2) The second one involves a finite-to-one continuous endomorphism $r$ on a compact metric space $X$.

We shall refer to (1) as the IFS case and to (2) as the endomorphism case.

In both situations we will be interested in random walks on the branches $\tau_{i}$ (see, e.g., Jo05a ). When the endomorphism $r$ is given, the branches are determined by an enumeration of the inverse images, i.e., $r\left(\tau_{i}(x)\right)=x$. When we are dealing with a general IFS, the endomorphism is not given a priori, and in some cases it might not even exist (for example, when the IFS has overlaps).

We will be interested in the Ruelle operator associated to these random walks and some nonnegative weight function $W$ on $X$ :

$$
R_{W} f(x)=\sum_{i=1}^{N} W\left(\tau_{i} x\right) f\left(\tau_{i} x\right),
$$

in the case of an IFS, or

$$
R_{W} f(x)=\sum_{y \in r^{-1}(x)} W(y) f(y)
$$

in the case of an endomorphism $r$. In some instances, multiplicity has to be counted, such as in the case of a rational map on the Julia set (see [Bea91, Bro65, and Mane).

4.1. Harmonic functions. In this section we will study the eigenvalue problem $R_{W} h=h$ in both of the cases for the operator $R_{W}$, i.e., both for the general case (4.1) and the special case (4.2) of IFSs. There is a substantial literature on the harmonic analysis of $R_{W}$; see, e.g., [AtNe04. Here we will focus mainly on the connection between $R_{W}$ and the problem of finding orthonormal bases (ONBs); see JoPe98] and BrJo99].

We make the convention to use the same notation

$$
\sum_{y \in r^{-1}(x)} f(y):=\sum_{i=1}^{N} f\left(\tau_{i} x\right),
$$

for slightly different context, even in the case of an IFS when $r$ is not really defined.

In the case of an IFS, we will denote by $r^{-n}(x)$ the set

$$
r^{-n}(x):=\left\{\tau_{\omega_{1}} \cdots \tau_{\omega_{n}} x \mid \omega_{1}, \ldots, \omega_{n} \in\{1, \ldots, N\}\right\} .
$$

The analysis of the harmonic functions for these operators, i.e., the functions $R_{W} h=h$, involves the construction of certain probability measures on the set of paths. These constructions and their properties are given in detail in Jo05a, DuJo04a, DuJo05] and DuJo04b. Here we recall the main ingredients.

For every point $x$ in $X$, we define a path starting at $x$ to be a finite or infinite sequence of points $\left(z_{1}, z_{2}, \ldots\right)$ such that $r\left(z_{1}\right)=x$ and $r\left(z_{n+1}\right)=z_{n}$ for all $n$. In the case of an IFS when $r$ is not given, a path is a sequence of letters $\left(\omega_{1}, \omega_{2}, \ldots\right)$ in the alphabet $\{1, \ldots, N\}$. These sequences can be identified with $\left(\tau_{\omega_{1}} x, \tau_{\omega_{2}} \tau_{\omega_{1}} x, \ldots, \tau_{\omega_{n}} \cdots \tau_{\omega_{1}} x, \ldots\right)$. We denote by $\Omega_{x}$ the set of infinite paths 
starting at $x$. We denote by $\Omega_{x}^{(n)}$ the set of paths of length $n$ starting at $x$. We denote by $X_{\infty}$ the set of all infinite paths starting at any point in $X$.

For a nonnegative function $W$ on $X$ such that

$$
\sum_{y \in r^{-1}(x)} W(y)=1 \quad \text { or } \quad \sum_{i=1}^{N} W\left(\tau_{i} x\right)=1,
$$

and following Kolmogorov, one can define probability measures $P_{x}$ on $\Omega_{x}, x \in X$, such that, for a function $f$ on $\Omega_{x}$ which depends only on the first $n+1$ coordinates,

$$
P_{x}(f)=\sum_{\left(z_{1}, \ldots, z_{n}\right) \in \Omega_{x}^{(n)}} W\left(z_{1}\right) W\left(z_{2}\right) \cdots W\left(z_{n}\right) f\left(z_{1}, \ldots, z_{n}\right),
$$

which in the case of an IFS has the meaning

$$
P_{x}(f)=\sum_{\omega_{1}, \ldots, \omega_{n}} W\left(\tau_{\omega_{1}} x\right) W\left(\tau_{\omega_{2}} \tau_{\omega_{1}} x\right) \cdots W\left(\tau_{\omega_{n}} \cdots \tau_{\omega_{1}} x\right) f\left(\omega_{1}, \ldots, \omega_{n}\right) .
$$

The connection between $P_{x}$ and $R_{W}$ is given as follows. Let $F \in C(X)$, and set

$$
f_{n}\left(\omega_{1}, \ldots, \omega_{n}\right):=F\left(\tau_{\omega_{n}} \cdots \tau_{\omega_{1}} x\right) .
$$

Then

$$
P_{x}\left(f_{n}\right)=R_{W}^{n}(F)(x)
$$

Next, we define a cocycle to be a function $V$ on $X_{\infty}$ such that for any path $\left(z_{1}, z_{2}, \ldots\right)$

$$
V\left(z_{1}, z_{2}, \ldots\right)=V\left(z_{2}, z_{3}, \ldots\right)
$$

for an IFS, this rewrites as

$$
V\left(x, \omega_{1}, \omega_{2}, \ldots\right)=V\left(\tau_{\omega_{1}} x, \omega_{2}, \ldots\right) .
$$

The main result we need here is that there is a one-to-one correspondence between bounded cocycles and bounded harmonic functions for $R_{W}$. The correspondence is given by:

Theorem 4.1. Let $W$ be a nonnegative measurable function on $X$ with $R_{W} 1=1$.

(i) If $V$ is a bounded, measurable cocycle on $\Omega$, then the function $h$ defined by

$$
h(x)=\int_{\Omega_{x}} V\left(\left(z_{n}\right)_{n \geq 1}\right) d P_{x}\left(\left(z_{n}\right)_{n \geq 1}\right), \quad x \in X,
$$

is a bounded harmonic function, i.e., $R_{W} h=h$.

(ii) If $h$ is a bounded harmonic function for $R_{W}$, then for every $x$, the limit

$$
V\left(\left(z_{n}\right)_{n \geq 1}\right):=\lim _{n \rightarrow \infty} h\left(z_{n}\right)
$$

exists for $P_{x}$ almost every path $\left(z_{n}\right)_{n \geq 0}$ that starts at $x$, and it defines a cocycle. Moreover, the equation in (ii) holds for this $V$.

Proof. We only sketch the idea for the proof to include the case of overlapping IFSs. The details are contained in [Jo05a, DuJo04a], DuJo05] and [AtNe04]. (1i) is the result of a computation; see Section 2.7 of [Jo05a] and Corollary 7.3 in [DuJo04a]. For (iii) we use martingales. For each $n$ denote by $\mathfrak{B}_{n}$, the sigma algebras generated by all $n$-cylinders in $\Omega_{x}$. The map $\left(z_{n}\right)_{n \geq 1} \mapsto h\left(z_{n}\right)$ can be seen to be a bounded martingale with respect to these sigma algebras and the measure $P_{x}$. Then Doob's martingale theorem implies the convergence in (iii). The fact that the limit $V$ is a cocycle follows again by computation (see the results mentioned before). 
4.2. Lifting the IFS case to the endomorphism case. Now consider an IFS $\left(X,\left(\tau_{i}\right)_{i=1}^{N}\right)$ where the maps $\tau_{i}$ are contractions. The application $\pi$ from the symbolic model $\Omega$ to the attractor $X$ of the IFS is given by

$$
\pi\left(\omega_{1}, \omega_{2}, \ldots\right)=\lim _{n \rightarrow \infty} \tau_{\omega_{1}} \tau_{\omega_{2}} \cdots \tau_{\omega_{n}} x_{0},
$$

where $x_{0}$ is some arbitrary point in $X$.

The map $\pi$ is continuous and onto; see Hut81 and YHK97. We will use it to lift the elements associated to the IFS, up from $X$ to $\Omega$, which is endowed with the endomorphism given by the shift $r_{\Omega}$. The inverse branches of $r_{\Omega}$ are

$$
\tilde{\tau}_{i}(\omega)=i \omega \quad(\omega \in \Omega)
$$

where, if $\omega=\left(\omega_{1}, \omega_{2}, \ldots\right)$, then $i \omega=\left(i, \omega_{1}, \omega_{2}, \ldots\right)$. This process serves to erase the overlap between the different sets $\tau_{i}(X)$.

The next lemma requires just some elementary computations.

Lemma 4.2. For a function $W$ on $X$ denote by $\tilde{W}:=W \circ \pi$.

(i) If $R_{W} 1=1$, then $R_{\tilde{W}} 1=1$.

(ii) For a function $f$ on $X, R_{\tilde{W}}(f \circ \pi)=\left(R_{W} f\right) \circ \pi$.

(iii) For a function $h$ on $X, R_{W} h=h$ if and only if $R_{\tilde{W}}(h \circ \pi)=h \circ \pi$.

(iv) If $\tilde{\nu}$ is a measure on $\Omega$ such that $\tilde{\nu} \circ R_{\tilde{W}}=\tilde{\nu}$, then the measure $\nu$ on $X$ defined by $\nu(f)=\tilde{\nu}(f \circ \pi)$, for $f \in C(X)$, satisfies $\nu \circ R_{W}=\nu$.

Lemma 4.3. If $W$ is continuous, nonnegative function on $X$ such that $R_{W} 1=1$, and if $\nu$ is a probability measure on $X$ such that $\nu \circ R_{W}=\nu$, then there exists a probability measure $\tilde{\nu}$ on $\Omega$ such that $\tilde{\nu} \circ R_{\tilde{W}}=\tilde{\nu}$ and $\tilde{\nu}(f \circ \pi)=\nu(f)$ for all $f \in C(X)$.

Proof. Consider the set

$$
\tilde{M}_{\nu}:=\left\{\tilde{\nu} \mid \tilde{\nu} \text { is a probability measure on } \Omega, \tilde{\nu} \circ \pi^{-1}=\nu\right\} .
$$

First, we show that this set is nonempty. For this, define the linear functional $\Lambda$ on the space $\{f \circ \pi \mid f \in C(X)\}$ by $\Lambda(f \circ \pi)=\nu(f)$, for $f \in C(X)$. This is well defined, because $\pi$ is surjective. It is also continuous, and it has norm 1. Using Hahn-Banach's theorem, we can construct an extension $\tilde{\nu}$ of $\Lambda$ to $C(\Omega)$ such that $\|\tilde{\nu}\|=1$. But we also have $\tilde{\nu}(1)=\nu(1)=1$, and this implies that $\tilde{\nu}$ is positive (see [Rud87]), so it is an element of $\tilde{M}_{\nu}$.

By Alaoglu's theorem, $\tilde{M}_{\nu}$ is weakly compact and convex. Consider the map $\tilde{\nu} \mapsto \tilde{\nu} \circ R_{\tilde{W}}$. It is continuous in the weak topology, because $R_{\tilde{W}}$ preserves continuous functions. Also, if $\tilde{\nu}$ is in $\tilde{M}_{\nu}$, then

$$
\tilde{\nu} \circ R_{\tilde{W}}(f)=\tilde{\nu}\left(R_{\tilde{W}}(f \circ \pi)\right)=\tilde{\nu}\left(\left(R_{W} f\right) \circ \pi\right)=\nu\left(R_{W} f\right)=\nu(f),
$$

so $\tilde{\nu} R_{\tilde{W}}$ is again in $\tilde{M}_{\nu}$. We can apply the Markov-Kakutani fixed point theorem Rud91 to obtain the conclusion. 


\section{A positive EIGENVALUE}

When the system $(X, r, W)$ is given as above, then the corresponding Ruelle operator $R_{W}$ of (2.1) is positive in the sense that it maps positive functions to positive functions. (By positive, we mean pointwise nonnegative. This will be the context below, and the term "strictly positive" will be reserved if we wish to exclude the zero case.) In a number of earlier studies Mane, Rue89, strict positivity has been assumed for the function $W$, but for the applications that interest us here (such as wavelets and fractals), it is necessary to allow functions $W$ that have nontrivial zero-sets, i.e., which are not assumed strictly positive.

A basic idea in the subject is that the study of spectral theory for $R_{W}$ is in a number of ways analogous to that of the familiar special case of positive matrices studied first by Perron and Frobenius. A matrix is said to be positive if its entries are positive. Motivated by the idea of Perron and Frobenius we begin with a lemma which shows that many spectral problems corresponding to a positive eigenvalue $\lambda$ can be reduced to the case $\lambda=1$ by a simple renormalization.

Lemma 5.1. Assume that the inverse orbit of any point under $r^{-1}$ is dense in $X$. Suppose also that there exists $\lambda_{m}>0$ and $h_{m}$ positive, bounded and bounded away from zero, such that

$$
R_{W} h_{m}=\lambda_{m} h_{m}
$$

Define

$$
\tilde{W}:=W \frac{h_{m}}{\lambda_{m} h_{m} \circ r} .
$$

Then

(i) $\lambda_{m} R_{\tilde{W}}=M_{h_{m}}^{-1} R_{W} M_{h_{m}}$, where $M_{h_{m}} f=h_{m} f$;

(ii) $R_{\tilde{W}} 1=1$;

(iii) $\lambda_{m} R_{\tilde{W}}$ and $R_{W}$ have the same spectrum;

(iv) $R_{W} h=\lambda h$ iff $R_{\tilde{W}}\left(h_{m}^{-1} h\right)=\frac{\lambda}{\lambda_{m}} h_{m}^{-1} h$;

(v) If $\nu$ is a measure on $X, \nu\left(R_{W} g\right)=\lambda \nu(g)$ for all $g \in C(X)$ iff $\nu\left(h_{m} R_{\tilde{W}} g\right)=$ $\frac{\lambda}{\lambda_{m}} \nu\left(h_{m} g\right)$ for all $g \in C(X)$.

With this lemma, we will consider from now on the cases when $R_{W} 1=1$.

Remark 5.2. We now turn to the study of

$$
H_{W}(1):=\left\{h \in C(X) \mid R_{W} h=h\right\} .
$$

By analogy to the classical theory, we expect that the functions $h$ in $H_{W}(1)$ have small zero sets. A technical condition is given in Proposition 5.8 which implies that if $h$ is nonconstant in $H_{W}(1)$, then its zeroes are contained in the union of the $W$-cycles.

Proposition 5.3. Let $W$ be continuous with $R_{W} 1=1$ and suppose $R_{W} f$ is continuous whenever $f$ is. Then the set

$$
M_{\mathrm{inv}}:=\left\{\nu \mid \nu \text { is a probability measure on } X, \nu \circ R_{W}=\nu\right\}
$$

is a nonempty convex set, compact in the weak topology. In the case of an endomorphism, if $\nu \in M_{\mathrm{inv}}$, then $\nu=\nu \circ r^{-1}$. The extreme points of $M_{\mathrm{inv}}$ are the ergodic invariant measures. 
Proof. The operator $\nu \mapsto \nu \circ R_{W}$ maps the set of probability measures to itself, and is continuous in the weak topology. The fact that $M_{\mathrm{inv}}$ is nonempty follows from the Markov-Kakutani fixed point theorem [Rud91]. The set is clearly convex, and it is compact due to Alaoglu's theorem. If $\nu \in M_{\mathrm{inv}}$ then

$$
\nu(f)=\nu\left(f R_{W} 1\right)=\nu\left(R_{W}(f \circ r)\right)=\nu(f \circ r), \quad f \in C(X) .
$$

Now, in the case of an endomorphism, if $\nu$ is an extreme point for $M_{\text {inv }}$ and if it is not ergodic, then there is a subset $A$ of $X$ such that $r^{-1}(A)=A$ and $0<\nu(A)<1$. Then define the measure $\nu_{A}$ by

$$
\nu_{A}(E)=\nu(E \cap A) / \nu(A), \quad E \text { measurable, }
$$

and similarly $\nu_{X \backslash A}$. Then $\nu=\nu(A) \nu_{A}+(1-\nu(A)) \nu_{X \backslash A}$. Also $\nu_{A}$ and $\nu_{X \backslash A}$ are in $M_{\text {inv }}$ because, for $f \in C(X)$,

$$
\begin{aligned}
\int_{X} R_{W} f d \nu_{A} & =\frac{1}{\nu(A)} \int_{X} \chi_{A} R_{W} f d \nu=\frac{1}{\nu(A)} \int_{X} R_{W}\left(\chi_{A} \circ r f\right) d \nu \\
& =\frac{1}{\nu(A)} \int_{X} R_{W}\left(\chi_{A} f\right) d \nu=\frac{1}{\nu(A)} \int_{X} \chi_{A} f d \nu=\int_{X} f d \nu_{A} .
\end{aligned}
$$

This contradicts the fact that $\nu$ is an extreme point. Conversely, if $\nu$ is ergodic, then if $\nu=\lambda \nu_{1}+(1-\lambda) \nu_{2}$ with $0<\lambda<1$ and $\nu_{1}, \nu_{2} \in M_{\text {inv }}$, then $\nu_{1}$ and $\nu_{2}$ are absolutely continuous with respect to $\nu$. Let $f_{1}, f_{2}$ be the Radon-Nikodym derivatives. We have that $\lambda f_{1}+(1-\lambda) f_{2}=1, \nu$-a.e. Since $\nu, \nu_{1}$, and $\nu_{2}$ are all in $M_{\text {inv }}$, we get that

$$
\nu\left(f f_{1} \circ r\right)=\nu\left(R_{W}\left(f f_{1} \circ r\right)\right)=\nu\left(f_{1} R_{W} f\right)=\nu_{1}\left(R_{W} f\right)=\nu_{1}(f)=\nu\left(f f_{1}\right) .
$$

Therefore $f_{1}=f_{1} \circ r, \nu$-a.e. But as $\nu$ is ergodic, $f_{1}$ is constant $\nu$-a.e; similarly for $f_{2}$. This and the fact that the measures are probability measures, implies that $f_{1}=f_{2}=1$, so $\nu=\nu_{1}=\nu_{2}$, and $\nu$ is extreme.

Theorem 5.4. Assume that the inverse orbit of every point $x \in X, O^{-}(x)=$ $\left\{y \in X \mid y \in r^{-n}(x)\right.$, for some $\left.n \in \mathbb{N}\right\}$ is dense in $X$. Let $W \in C(X)$ (or $W \circ \pi$ in the IFS case) have finitely many zeroes. Suppose $R_{W} 1=1$. Let $\nu$ be a probability measure with $\nu \circ R_{W}=\nu$. Then either $\nu$ has full support, or $\nu$ is atomic and supported on $W$-cycles.

Proof. First consider the case of an endomorphism $r$. Suppose that the support of $\nu$ is not full, so there exists a nonempty open set $U$ with $\nu(U)=0$. Denote by $E$ the smallest completely invariant subset of $X$ that contains the zeroes of $W$ :

$$
E=\bigcup_{m, n \geq 0} r^{-m}\left(r^{n}(\operatorname{zeroes}(W))\right) .
$$

Note that

$$
r^{n}(A \backslash E)=r^{n}(A) \backslash E, \quad n \geq 0, A \subset X .
$$

We have

$$
\nu\left(R_{W} \chi_{U \backslash E}\right)(x)=\int_{X} \sum_{y \in r^{-1}(x)} W(y) \chi_{U \backslash E}(y) d \nu(x)=\int_{X} \chi_{U \backslash E}(x) d \nu(x)=0 .
$$

Therefore, since $W$ is positive on $X \backslash E$, and since $y \in U \backslash E$ iff $x \in r(U \backslash E)$, it follows that $\nu(r(U \backslash E))=0$. By induction $\nu\left(r^{n}(U \backslash E)\right)=0$ for all $n$. 
However, since the inverse orbit of every point is dense in $X$, we have that $\bigcup_{n} r^{n}(U)=X$. With equation (5.1), we get that $\bigcup_{n} r^{n}(U \backslash E)=X \backslash E$. In conclusion, $\nu$ has to be supported on $E$.

Now $E$ is countable, hence there must be a point $x_{0} \in E$ such that $\nu\left(\left\{x_{0}\right\}\right)>0$.

Using the invariance, we obtain

$$
\begin{aligned}
0<\nu\left(\left\{x_{0}\right\}\right) & =\nu\left(R_{W} \chi_{x_{0}}\right) \\
& =\int_{X} \sum_{y \in r^{-1}(x)} W(y) \chi_{x_{0}}(y) d \nu(x)=W\left(x_{0}\right) \nu\left(\left\{r\left(x_{0}\right)\right\}\right) .
\end{aligned}
$$

Since $R_{W} 1=1$, we have $W\left(x_{0}\right) \leq 1$, so $\nu\left(\left\{x_{0}\right\}\right) \leq \nu\left(\left\{r\left(x_{0}\right)\right\}\right)$. By induction, we obtain

$$
0<\nu\left(\left\{x_{0}\right\}\right) \leq \nu\left(\left\{r\left(x_{0}\right\}\right) \leq \cdots \leq \nu\left(\left\{r^{n}\left(x_{0}\right)\right\}\right) \leq \cdots .\right.
$$

Also, since $\nu$ is $r$-invariant,

$$
\nu\left(r^{-n-1}\left(x_{0}\right)\right)=\nu\left(r^{-n}\left(x_{0}\right)\right)=\cdots=\nu\left(r^{-1}\left(x_{0}\right)\right)=\nu\left(\left\{x_{0}\right\}\right) .
$$

But the measure is finite so the sets $r^{-n}\left(x_{0}\right)$ must intersect, therefore, $x_{0}$ has to be a point in a cycle; so $r^{n}\left(x_{0}\right)=x_{0}$ for some $n \geq 1$. Hence we will have equality in (5.3). Looking at (5.2), we see that we must have $W\left(x_{0}\right)=1$, so $\left\{x_{0}, r\left(x_{0}\right), \ldots, r^{n-1}\left(x_{0}\right)\right\}$ indeed forms a $W$-cycle.

Now consider the case of an IFS. The function $\tilde{W}=W \circ \pi$ has finitely many zeroes. If $\nu$ is invariant, then by Lemma 4.3, there exists a measure $\tilde{\nu}$ on $\Omega$ which is invariant for $R_{\tilde{W}}$ and such that $\tilde{\nu} \circ \pi^{-1}=\nu$.

By the previous argument, $\tilde{\nu}$ has either full support or is supported on some $\tilde{W}$-cycles. If $\tilde{\nu}$ has full support, then for every nonempty open subset $U$ of $X$, $\tilde{\nu}\left(\pi^{-1}(U)\right)>0$, so $\nu(U)>0$. Therefore $\nu$ has full support.

If $\tilde{\nu}$ is supported on some union of cycles $\mathcal{C}:=\bigcup_{i} \tilde{C}_{i}$, then

$$
\nu(X \backslash \pi(\mathcal{C}))=\tilde{\nu}\left(\pi^{-1}(X \backslash \mathcal{C})\right) \leq \nu(\Omega \backslash \mathcal{C})=0 .
$$

So $\nu$ is supported on the union of cycles $\pi(\mathcal{C})$.

Proposition 5.5. If $W \in C(X), W \geq 0$, and $R_{W} 1=1$, and if $W$ has no cycles, then every invariant measure $\nu$ has no atoms.

Proof. The argument needed is already contained in the proof of Theorem 5.4 see the inequality (5.2) and the next few lines after it.

In the next proposition we want to include the case of functions $W$ which may have infinitely many zeroes. This is why we define the following technical condition:

Definition 5.6. We say that a function $W$ on $X$ satisfies the transversality of the zeroes condition (TZ):

(i) If $x \in X$ is not a cycle, then there exists $n_{x} \geq 0$ such that, for $n \geq n_{x}$, $r^{-n}(x)$ does not contain any zeroes of $W$;

(ii) If $\left\{x_{0}, x_{1}, \ldots, x_{p}\right\}$ are on a cycle with $x_{1} \in r^{-1}\left(x_{0}\right)$, then every $y \in r^{-1}\left(x_{0}\right)$, $y \neq x_{1}$ is either not on a cycle, or $W(y)=0$.

Proposition 5.7. Suppose the inverse orbit of every point is dense in $X, W$ is continuous, it satisfies the $T Z$ condition, and $R_{W} 1=1$. If

$$
\operatorname{dim}\left\{h \in C(X) \mid R_{W} h=h\right\} \geq 1,
$$

then there exist $W$-cycles. 
Proof. Take $h$ a nonconstant function in $C(X)$ with $R_{W} h=h$. Then the function $\left\|\frac{h+\bar{h}}{2}\right\|_{\infty}-\left(\frac{h+\bar{h}}{2}\right)$ is again a continuous function, it is fixed by $R_{W}$, nonnegative, and it has some zeroes. We relabel this function by $h$. Let $z_{0} \in X$ be a zero of $h$. Then

$$
\sum_{y \in r^{-1}\left(z_{0}\right)} W(y) h(y)=h\left(z_{0}\right)=0,
$$

therefore, for all $y \in r^{-1}\left(z_{0}\right)$, we have $W(y)=0$, or $h(y)=0$. We cannot have $W(y)=0$ for all such $y$, because this would contradict $R_{W} 1=1$. Thus there is some $z_{1} \in r^{-1}\left(z_{0}\right)$, with $h\left(z_{1}\right)=0$ and $W\left(z_{1}\right) \neq 0$. Inductively, we can find a sequence $z_{n}$ such that $z_{n+1} \in r^{-1}\left(z_{n}\right), W\left(z_{n}\right) \neq 0$ and $h\left(z_{n}\right)=0$.

We want to prove that $z_{0}$ is a point of a cycle. Suppose not. Then for $n$ big enough, there are no zeroes of $W$ in $r^{-n}\left(z_{0}\right)$. But then look at $z_{n}$ : using the equation $R_{W} h\left(z_{n}\right)=h\left(z_{n}\right)$, we obtain that $h$ is 0 on $r^{-1}\left(z_{n}\right)$. By induction, we get that $h$ is 0 on $r^{-k}\left(z_{n}\right)$ for all $k \in \mathbb{N}$. Since the inverse orbit of $z_{n}$ is dense, this implies that $h$ is constant 0 . This contradiction shows that $z_{0}$ is a point of some cycle, so every zero of $h$ lies on a cycle. But then $z_{1}$ is a point in the same cycle (because of the TZ condition and the fact that $z_{1}$ is on some cycle and $W\left(z_{1}\right) \neq 0$ ). Also,

$$
\sum_{y \in r^{-1}\left(z_{0}\right)} W(y) h(y)=h\left(z_{0}\right)=0,
$$

and, if $y \in r^{-1}\left(z_{0}\right), y \neq z_{1}$, then $y$ is not a point of a cycle so it cannot be a zero for $h$. Therefore $W(y)=0$, so $W\left(z_{1}\right)=1$. Since this can be done for all points $z_{i}$, this implies that the cycle is a $W$-cycle.

The proof of Proposition 5.7 can be used to obtain the following:

Proposition 5.8. Assume $W$ is continuous and satisfies the $T Z$ condition. Let $h \in C(X)$ be nonnegative and $R_{W} h=h$. Then either there exists some $x \in X$ such that $h$ is constant 0 on $O^{-}(x)$, or all the zeroes of $h$ are points on some $W$-cycle.

Proposition 5.9. Suppose $W$ is as before. In the case of an endomorphism system $(X, r)$, if $\nu$ is an extremal invariant state, $\nu \circ R_{W}=\nu, h \in C(X)$ and $R_{W} h=h$, then $h$ is constant $\nu$-a.e.

Proof. If $\nu$ is extremal, then $\nu$ is ergodic with respect to $r$. We have for all $f \in$ $C(X)$,

$$
\nu(f h)=\nu\left(f R_{W} h\right)=\nu\left(R_{W}(f \circ r h)\right)=\nu(f \circ r h)=\cdots=\nu\left(f \circ r^{n} h\right) .
$$

We can apply Birkhoff's theorem and Lebesgue's dominated convergence theorem to obtain that

$$
\nu(f h)=\lim _{n \rightarrow \infty} \nu\left(\frac{1}{n} \sum_{k=0}^{n-1} f \circ r^{k} h\right)=\nu(\nu(f) h)=\nu(f \nu(h)) .
$$

Thus $\nu(h)=h, \nu$-a.e. 
Theorem 5.10. Suppose $W \in C(X), R_{W} 1=1$, the inverse orbit of any point is dense in $X$, and there are no $W$-cycles.

(i) In the case of an endomorphism system $(X, r)$, if $W$ has finitely many zeroes and $R_{W}: C(X) \rightarrow C(X)$ has an eigenvalue $\lambda \neq 1$ of absolute value 1 then, if $h \in C(X)$ and $R h=\lambda h$, then $h=\lambda h \circ r$.

If in addition $r$ has at least one periodic orbit, then $\lambda$ is a root of unity. If $\lambda^{p}=1$ with $p$ the smallest with this property, then there exists a partition of $X$ into disjoint compact open sets $A_{k}, k \in\{0, \ldots, p-1\}$, such that $r\left(A_{k}\right)=A_{k+1}(k \in\{0, \ldots, p-2\}), r\left(A_{p-1}\right)=A_{0}, h$ is constant $h_{k}$ on $A_{k}$, and $h_{k}=\lambda h_{k+1}, k \in\{0, \ldots, p-2\}$.

(ii) In the case of an IFS, if $W \circ \pi$ has finitely many zeroes, there are no $\lambda \neq 1$ with $|\lambda|=1$ such that $R_{W} h=\lambda h$ for $h \neq 0, h \in C(X)$, i.e., $R_{W}$ has no peripheral spectrum as an operator in $C(X)$, other than $\lambda=1$.

Proof. (ii) Suppose $|\lambda|=1, \lambda \neq 1$, and there is $h \in C(X) h \neq 0$ such that $R_{W} h=$ $\lambda h$. Then we have

$$
|h(x)|=\left|R_{W} h(x)\right|=\left|\sum_{y \in r^{-1}(x)} W(y) h(y)\right| \leq R_{W}|h|(x), \quad x \in X .
$$

By Proposition 5.3, there is an extremal invariant measure $\nu$. We have

$$
\nu(|h|) \leq \nu\left(R_{W}|h|\right)=\nu(|h|) .
$$

Thus we have equality in (5.5), and since the support of $\nu$ is full (Theorem 5.4) and the functions are continuous, it follows that $|h|=R_{W}|h|$. Using Proposition [5.9, we get that $|h|$ is a constant, and we may take $|h|=1$. But then, we have equality in

$$
|h|=\left|R_{W}(h)\right| \leq R_{W}(|h|),
$$

and this implies that, for all $x \in X$, the numbers $W(y) h(y)$ for $y \in r^{-1}(x)$ are proportional, i.e., there is a complex number $c(x)$ with $|c(x)|=1$, and some nonnegative numbers $a_{y} \geq 0\left(y \in r^{-1}(x)\right)$ such that $W(y) h(y)=c(x) a_{y}$. Since $|h|=1$, we obtain that $W(y)=a_{y}$ and $h(y)=c(x)$. Thus $h$ is constant on the roots of $x$, and moreover $h(y)=c(r(y))$, for all $y \in X$. But then

$$
\lambda c \circ r=\lambda h=R_{W} h=R_{W}(c \circ r)=c R_{W}(1)=c,
$$

so $h=c \circ r=\lambda c \circ r \circ r=\lambda h \circ r$.

Let $x_{0}$ be a periodic point for $r$ of period $n$. Then $c\left(x_{0}\right)=\lambda^{n} c\left(r^{n}\left(x_{0}\right)\right)=\lambda^{n} c\left(x_{0}\right)$, therefore $\lambda^{n}$ is a root of unity. Take $p \geq 2$, the smallest positive integer with $\lambda^{p}=1$.

If $I_{k}:=\left\{e^{2 \pi i \theta} \mid \theta \in[k / p,(k+1) / p)\right\}$, then note that $\lambda^{-1} I_{k}=I_{\sigma(k)}$ for some cyclic permutation $\sigma$ of $\{0, \ldots, p-1\}$. Denote by $A_{k}$ the set

$$
A_{k}:=\left\{x \in X \mid c(x) \in I_{\sigma^{k}(0)}\right\}, \quad k \in\{0, \ldots, p\} .
$$

Then the sets $\left(A_{k}\right)_{k=0, \ldots, p-1}$ are disjoint, they cover $X, A_{p}=A_{0}$, and the relation $\lambda c \circ r=c$ implies that $r$ maps $A_{k}$ onto $A_{k+1}$.

So each set $A_{k}$ is invariant for $r^{p}$. Next we claim that $r^{p}$ restricted to $A_{k}$ is ergodic. If not, there exists a subset $A$ of $A_{k}$ which is completely invariant for $r^{p}$ and $0<\nu(A)<\nu\left(A_{k}\right)$. But then consider the set

$$
B=A \cup r^{-1}(A) \cup \cdots \cup r^{-(p-1)}(A) .
$$

The set $B$ is completely invariant for $r$, and $0<\nu(B) \leq 1-\nu\left(A_{k} \backslash A\right)<1$, which contradicts the fact that $\nu$ is ergodic with respect to $r$. 
Thus we have $r^{p}$ ergodic on $A_{k}$, and $c \circ r^{p}=c$. This implies that $c$ is constant $c_{k}$ on $A_{k}$. The constants are related by $c_{k}=\lambda^{-k} c_{0}$. Moreover, $A_{k}=c^{-1}\left(c_{k}\right)$, so $A_{k}$ is compact and open. With $h=c \circ r$, this gives us the desired result.

(iii) In the case of an IFS, suppose $R_{W} h=\lambda h$ as in the hypothesis. Then, lifting to $\Omega$ we get $R_{\tilde{W}}(h \circ \pi)=\lambda h \circ \pi$. However, $r_{\Omega}$ has a fixed point $\omega=(1,1, \ldots)$. Therefore, (ii) implies that $h \circ \pi$ is constant, so $h$ is constant also.

Remark 5.11. The existence of a periodic point is required to guarantee the fact that $\lambda$ is a root of unity. Here is an example when $\lambda$ can be an irrational rotation. Take the map $z \mapsto \lambda^{-1} z$ on the unit circle $\mathbb{T}$, and take $h(z)=z$. It satisfies $h=\lambda h \circ r$. The inverse orbits are clearly dense.

Another example, which is not injective, is the following: take some dynamical system $g: Y \rightarrow Y$ which has some strong mixing properties. For example $Y=\mathbb{T}$ and $g(z)=z^{N}$. Then define $r$ on $\mathbb{T} \times X$ by $r(z, x)=\left(\lambda^{-1} z, g(x)\right)$, and define $c(z, y)=z$. The strong mixing properties are necessary to obtain the density of the inverse orbits. We check this for $g(z)=z^{N}$.

Take $z_{0}, z_{1} \in \mathbb{T}, y_{0}, y_{1} \in \mathbb{T}$. Fix $\epsilon>0$. There exists $n$ as large as we want such that $\left|\lambda^{n} z_{0}-z_{1}\right|<\epsilon / 2$. Note that $g^{-n}\left(y_{0}\right)$ contains $N^{n}$ points such that any point in $\mathbb{T}$ is at a distance less than $2 \pi / N^{n}$ from one of these points. In particular, there is $w_{0}$ with $g^{n}\left(w_{0}\right)=y_{0}$ such that $\left|w_{0}-y_{1}\right|<\epsilon / 2$. This proves that the inverse orbit of $\left(z_{0}, y_{0}\right)$ is dense in $\mathbb{T} \times Y$.

For the dynamical systems we are interested in, the existence of a periodic point is automatic. That is why we will not be concerned about this case when $\lambda$ is an irrational rotation.

Corollary 5.12. Let $\sigma_{A}$ on $\Sigma_{A}$ be a subshift of finite type with irreducible matrix $A$, and let $W$ be a continuous function with $R_{W} 1=1$ and no $W$-cycles. Then 1 is the only eigenvalue for $R_{W}$ of absolute value 1 if and only if $A$ is aperiodic. When $A$ is periodic, of period $q$, the eigenvalues $\lambda$ of $R_{W}$ with $|\lambda|=1$ are roots $\left\{\lambda \mid \lambda^{q}=1\right\}$. There exists a partition $S_{0}, \ldots, S_{q-1}$ of $\{1, \ldots, N\}$ such that for all $i \in S_{k}, A_{i j}=1$ implies $j \in S_{k+1}, k \in\{0, \ldots, q-1\}\left(S_{q+1}:=S_{0}\right)$. For a $\lambda$ with $\lambda^{p}=1$, every continuous function $h$ with $R_{W} h=\lambda h$ is of the form

$$
h=\sum_{k=0}^{q-1} a \lambda^{-k} \chi_{\left\{\left(x_{n}\right)_{n} \in \Sigma_{A} \mid x_{0} \in S_{k}\right\}},
$$

where $a \in \mathbb{C}$.

Proof. If $A$ is aperiodic, it follows that, for every $k \in\{1, \ldots, N\}$ the greatest common divisor of the lengths of the periodic points that start with $k$ is 1 (see DGS76, Chapter 8]). But then, with Theorem [5.10, this means that $\lambda$ has to be 1.

If $A$ has period $q$, then with Proposition 8.15 in [DGS76], we can find the partition $\left(S_{k}\right)_{k=1, \ldots, q}$. Moreover, we have that the greatest common divisor of the lengths of the periodic orbits is $q$. Plugging the periodic points into the relation $h=\lambda h \circ r$ given by Theorem 5.10 , we obtain that $\lambda^{q}=1$. Therefore $q$ is a multiple of the order of $\lambda$ which we denote by $p$. Theorem 5.10 then yields a partition $\left(A_{k}\right)_{k \in\{0, \ldots, p-1\}}$ of $\Sigma_{A}$ with each $A_{k}$ compact, open, and invariant for $r^{p}$, hence also for $r^{q}$.

Denote by $\mathcal{S}_{k}$ the set $\mathcal{S}_{k}:=\left\{\left(x_{i}\right)_{i} \in \Sigma_{A} \mid x_{0} \in S_{k}\right\}$. It is clear that these sets are compact, open, and invariant for $r^{q}$ (actually $r\left(\mathcal{S}_{k}\right)=\mathcal{S}_{k+1}$ ). We claim that they 
are minimal with these properties. It is enough to prove this for $\mathcal{S}_{0}$. Indeed, if we take a small enough open subset of $\mathcal{S}_{0}$, we can assume it is a cylinder of the form

$$
C:=\left\{\left(x_{i}\right)_{i} \in \Sigma_{A} \mid x_{0}=a_{0}, \ldots, x_{n q}=a_{n q}\right\},
$$

for some fixed $a_{0}, \ldots, a_{n q}$. Then $a_{0} \in S_{0}, a_{1} \in S_{1}, \ldots, a_{n q} \in S_{n q}$. Take any $b \in S_{0}$. Since the matrix $A$ is irreducible, there exists an admissible path from $a_{n q}$ to $b$. Since $a_{n q}$ and $b$ are in $S_{0}$, the length of this path must be a multiple of $q$, say $m q$. But then $r^{(m+n) q}(C)$ will contain every infinite admissible word that starts with $b$. Since $b \in S_{0}$ was arbitrary, it follows that

$$
\bigcup_{m \geq 0} r^{m}(C)=\mathcal{S}_{0} .
$$

This proves the minimality of $\mathcal{S}_{0}$.

But for each $l \in\{0, \ldots, p-1\}, A_{l} \cap S_{k}$ is compact, open, and invariant for $r^{q}$, for all $k$. Therefore it is either empty or $S_{k}$. Hence, $A_{l}$ is a union of some of the sets $S_{k}$. The corollary follows from Theorem 5.10

Corollary 5.13. In the case of an endomorphism system $(X, r)$, assume there are no $W$-cycles, the inverse orbit of any point is dense in $X, W$ has finitely many zeroes, and $R_{W} 1=1$. If $X$ is connected, or if $r$ is topologically mixing, i.e., for every two nonempty open sets $U$ and $V$ there exists $n_{0} \geq 1$ such that $r^{-n}(U) \cap V \neq \emptyset$ for all $n \geq n_{0}$, then $R_{W}$ has no nontrivial eigenvalues of absolute value 1 . In particular, $r$ can be a rational map on a Julia set.

Proposition 5.14. In the case of an endomorphism system $(X, r)$, let $W, W^{\prime} \in$ $C(X), W, W^{\prime} \geq 0, R_{W} 1=R_{W^{\prime}} 1=1$. Suppose $\nu$ is an extreme point of the probability measures which are invariant for $R_{W}$, and similarly for $\nu^{\prime}$ and $R_{W^{\prime}}$. Then, if $\nu \neq \nu^{\prime}$, then $\nu$ and $\nu^{\prime}$ are mutually singular.

Proof. The fact that the measure are extremal implies that they are ergodic (Proposition 5.3.

Since $\nu$ and $\nu^{\prime}$ are ergodic and invariant for $r$, we can apply Birkhoff's theorem Yo98 to a continuous function $f$ such that $\nu(f) \neq \nu^{\prime}(f)$. We then have that

$$
\lim _{n \rightarrow \infty} \frac{1}{n} \sum_{k=0}^{n-1} f \circ r^{k}(x)=\nu(f), \quad \text { for } \nu \text {-a.e. } x
$$

and

$$
\lim _{n \rightarrow \infty} \frac{1}{n} \sum_{k=0}^{n-1} f \circ r^{k}(x)=\nu^{\prime}(f), \quad \text { for } \nu^{\prime} \text {-a.e. } x .
$$

But since $\nu(f) \neq \nu^{\prime}(f)$, this means that the measures are supported on disjoint sets, so they are mutually singular.

Corollary 5.15. Take $r(z)=z^{N}$ on $\mathbb{T}$. Suppose $W, W^{\prime} \in C(\mathbb{T})$ are Lipschitz, $R_{W} 1=R_{W^{\prime}} 1=1$, and suppose they have no cycles and they have finitely many zeroes. If $W \neq W^{\prime}$, then their invariant measures are mutually singular. In particular, if $W$ is not constant $\frac{1}{N}$, then $\nu$ is singular with respect to the Haar measure on $\mathbb{T}$.

Proof. The conditions in the hypothesis guarantee that the invariant measures are unique (see $\mathrm{Ba00}$ ), so extremality is automatic. The fact that $W \neq W^{\prime}$ insures that the measures $\nu$ and $\nu^{\prime}$ are different. The rest follows from Proposition 5.14 . 
When $m_{0}^{\prime}=\frac{1}{N}$, the invariant measure is the Haar measure.

Example 5.16 ([DuJo03]). Set $d=1, R=3$, and

$$
W(z):=\frac{1}{3}\left|\frac{1+z^{2}}{\sqrt{2}}\right|^{2}
$$

Then clearly $W(1)=2 / 3$, and $R_{W}$ satisfies $R_{W} 1=1$. The Perron-Frobenius measure $\nu_{W}$ is determined by $\nu_{W} R_{W}=\nu_{W}$ and $\nu_{W}(1)=1$.

Introducing the additive representation $\mathbb{T} \simeq \mathbb{R} / 2 \pi \mathbb{Z}$ via $z=e^{i t}$ we get

$$
W\left(e^{i t}\right)=\frac{2}{3} \cos ^{2}(t)
$$

and we checked in DuJo03 that the corresponding Perron-Frobenius measure $\nu_{W}$ is given by the classical Riesz product

$$
d \nu_{W}(t)=\frac{1}{2 \pi} \prod_{k=1}^{\infty}\left(1+\cos \left(2 \cdot 3^{k} t\right)\right) .
$$

It follows immediately from Corollary 5.15 that the measure $\nu_{W}$ representing the Riesz product has full support and is purely singular, conclusions which are not directly immediate.

Corollary 5.17 ([Ka48]; see also [BJP96]). Consider $\Omega:=\{1, \ldots, N\}^{\mathbb{N}}$, where $N \geq 2$ is an integer. For $p:=\left(p_{1}, p_{2}, \ldots, p_{N}\right)$ with $p_{i} \geq 0$ and $\sum_{i=1}^{N} p_{i}=1$, define the corresponding product measure $\mu_{p}$ on $\Omega$. Then, for $p \neq p^{\prime}$, the measures $\mu_{p}$ and $\mu_{p^{\prime}}$ are mutually singular.

Proof. Let $r=r_{\Omega}$ be the shift on $\Omega$. Define $W_{p}:=\sum_{i=1}^{N} p_{i} \chi_{\left\{\omega \mid \omega_{0}=i\right\}}$. Then it is easy to check (by analyzing cylinders) that $\mu_{p}$ is invariant for $R_{W_{p}}$. Also $R_{W_{p}} 1=1$, $W_{p}$ has no cycles, and it is Lipschitz. Therefore the invariant measure is unique, hence extremal, and the conclusion follows now from Proposition 5.14.

Remark 5.18. Note that the examples in Corollary 5.17 have no overlap. To illustrate the significance of overlap, it is interesting to compare with the family of Bernoulli convolutions. In this case $N=2$ and $p_{1}=p_{2}=\frac{1}{2}$, but the IFS varies with a parameter $\lambda$ as follows:

Let $\lambda \in(0,1)$. If we set $R:=\lambda^{-1}$ and $b_{ \pm}:= \pm \lambda^{-1}$, then we arrive at the IFS $\{\lambda x-1, \lambda x+1\}$. The corresponding measure $\mu_{\lambda}$ is the distribution of the random series $\sum_{n=0}^{\infty} \pm \lambda^{n}$ with the signs independently distributed with probability $\frac{1}{2}$, and Fourier transform

$$
\hat{\mu}_{\lambda}(t)=\prod_{n=0}^{\infty} \cos \left(2 \pi \lambda^{n} t\right), \quad t \in \mathbb{R} .
$$

The study of $\mu_{\lambda}$ for $\lambda \in(0,1)$ has a long history; see So95]. Solomyak proved that $\mu_{\lambda}$ has a density in $L^{2}$ for Lebesgue a.a. $\lambda \in\left(\frac{1}{2}, 1\right)$.

Set $\Omega=\prod_{0}^{\infty}\{-1,1\}$ and $B_{\lambda}=\left\{ \pm \lambda^{-1}\right\}$. Then one checks that the mapping $\pi_{\lambda}: \Omega \rightarrow X_{B_{\lambda}}$ from Definition 3.3 is $\pi_{\lambda}(\omega)=\sum_{k=0}^{\infty} \omega_{k} \lambda^{k}$. 


\section{The CASE OF CYCLES}

We will make the following assumptions:

$$
R_{W} 1=1
$$

$W$ satisfies the TZ condition in Definition 5.6.

In this section we will analyze the consequences of the existence of a $W$-cycle.

6.1. Harmonic functions associated with $W$-fixed points. Assume $x_{0}$ is a fixed point for $r$, i.e., $x_{0} \in r^{-1}\left(x_{0}\right)$, and the following condition is satisfied:

$$
W\left(x_{0}\right)=1 .
$$

Lemma 6.1. For $x \in X$ and $\left(z_{n}\right)_{n} \in \Omega_{x}$, the following relation holds:

$$
P_{x}\left(\left\{\left(z_{n}\right)_{n}\right\}\right)=\prod_{n=1}^{\infty} W\left(z_{n}\right) .
$$

Proof. The set $\left\{\left(z_{n}\right)_{n}\right\}$ can be written as the decreasing intersection of the cylinders $Z_{m}:=\left\{\left(\eta_{n}\right)_{n} \in \Omega_{x} \mid \eta_{k}=z_{k}\right.$, for $\left.k \leq m\right\}$. If we evaluate the measure of these cylinders we obtain

$$
P_{x}\left(Z_{m}\right)=W\left(z_{m}\right) W\left(z_{m-1}\right) \cdots W\left(z_{1}\right) .
$$

Taking the limit for $m \rightarrow \infty$, the lemma is proved.

For each $x$ in $X$ define the set

$$
\mathbf{N}_{x_{0}}(x):=\left\{\left(z_{n}\right)_{n} \in \Omega_{x} \mid \lim _{n \rightarrow \infty} z_{n}=x_{0}\right\} .
$$

Lemma 6.2. Define the function

$$
h_{x_{0}}(x):=P_{x}\left(\mathbf{N}_{x_{0}}(x)\right), \quad x \in X .
$$

Then $h_{x_{0}}$ is a nonnegative harmonic function for $R_{W}$, and $h_{x_{0}} \leq 1$.

Proof. Let $V_{x_{0}}(x, \omega)=\chi_{\mathbf{N}_{x_{0}}(x)}(\omega)$. It is then clear that $V_{x_{0}}$ is a cocycle. Since

$$
h_{x_{0}}(x)=P_{x}\left(\mathbf{N}_{x_{0}}(x)\right) \leq P_{x}(1)=1,
$$

Theorem 4.1 then implies that $h_{x_{0}}$ is a nonnegative harmonic function, and $h_{x_{0}} \leq$ 1.

Lemma 6.3. For the function $h_{x_{0}}$ in (6.5), the following equation holds:

$$
h_{x_{0}}\left(x_{0}\right)=1 \text {. }
$$

If $h$ is a nonnegative function with $R_{W} h=h, h\left(x_{0}\right)=1$, and $h$ is continuous at $x_{0}$, then $h_{x_{0}} \leq h$.

Proof. Using Lemma 6.1 and (6.3), we see that $P_{x_{0}}\left(\left\{\left(x_{0}, x_{0}, \ldots\right)\right\}\right)=1$. Therefore $h_{x_{0}}\left(x_{0}\right) \geq 1$, and with Lemma 6.2, we obtain that $h_{x_{0}}\left(x_{0}\right)=1$.

Take $x$ in $X$. For each path $\left(z_{n}\right)_{n=1}^{m}$ of length $m$ starting at $x$, choose an infinite path $\omega\left(\left(z_{n}\right)_{n \leq m}\right):=\left(z_{n}\right)_{n \geq 1}$ which starts with the given finite path and converges to $x$ (if such a path exists; if not, $\omega\left(\left(z_{n}\right)_{n \leq m}\right)$ is not defined). Let $Y_{m}$ be the set of all the chosen infinite paths, so

$$
Y_{m}:=\left\{\omega\left(\left(z_{n}\right)_{n \leq m}\right) \mid\left(z_{n}\right)_{n \leq m} \text { is a path that starts at } x\right\} .
$$


Next define $f_{m}: \mathbf{N}_{x_{0}}(x) \rightarrow \mathbb{C}$, by

$$
f_{m}\left(\left(z_{n}\right)_{n \geq 1}\right)= \begin{cases}W^{(m)}\left(z_{m}\right) h\left(z_{m}\right) & \text { if }\left(z_{n}\right)_{n \geq 1} \in Y_{m}, \\ 0 & \text { otherwise. }\end{cases}
$$

Then observe that

$$
\sum_{\mathbf{N}_{x_{0}}(x)} f_{m}\left(\left(z_{n}\right)_{n \geq 1}\right) \leq \sum_{r^{n}\left(z_{n}\right)=z_{0}} W^{(n)}\left(z_{n}\right) h\left(z_{n}\right)=\left(R_{W}^{n} h\right)(x)=h(x) .
$$

Also, because $h$ is continuous at $x_{0}$ and with Lemma 6.1, we get

$$
\lim _{m \rightarrow \infty} f_{m}\left(\left(z_{n}\right)_{n \geq 1}\right)=P_{x}\left(\left\{\left(z_{n}\right)_{n \geq 1}\right\}\right), \quad\left(z_{n}\right)_{n \geq 1} \in \mathbf{N}_{x_{0}}(x) .
$$

Now we can apply Fatou's lemma to the functions $f_{m}$ and, with (6.7) and (6.8), we obtain

$$
\begin{aligned}
h_{x_{0}}(x) & =\sum_{\left(z_{n}\right)_{n \geq 1} \in \mathbf{N}_{x_{0}}(x)} P_{x}\left(\left\{\left(z_{n}\right)_{n \geq 1}\right\}\right) \\
& =\sum_{\left(z_{n}\right)_{n \geq 1} \in \mathbf{N}_{x_{0}}(x)} \lim _{m \rightarrow \infty} f_{m}\left(\left(z_{n}\right)_{n \geq 1}\right) \\
& \leq \liminf _{m \rightarrow \infty} \sum_{\left(z_{n}\right)_{n \geq 1} \in \mathbf{N}_{x_{0}}(x)} f_{m}\left(\left(z_{n}\right)_{n \geq 1}\right) \leq h(x) .
\end{aligned}
$$

Definition 6.4. A fixed point $x_{0}$ is called repelling if there is $0<c<1$ and $\delta>0$ such that for all $x \in X$ with $d\left(x, x_{0}\right)<\delta$, there is a path $\left(z_{n}\right)_{n \geq 1}$ that starts at $x$ and such that $d\left(z_{n+1}, x_{0}\right) \leq c d\left(z_{n}, x\right)$ for all $n \geq 1$.

A cycle $C=\left\{x_{0}, \ldots, x_{p-1}\right\}$ is called repelling if each point $x_{i}$ is repelling for $r^{p}$, in the endomorphism case, or for the IFS $\left(\tau_{\omega_{1}} \cdots \tau_{\omega_{p}}\right)_{\omega_{1}, \ldots, \omega_{p}=1}^{N}$ in the IFS case.

Remark 6.5. In the case of an IFS, when the branches are contractive, each cycle is repelling. This is because, if $\tau_{\omega_{p-1}} \cdots \tau_{\omega_{0}}\left(x_{0}\right)=x_{0}$, then the repeated word $\left(\omega_{0}, \omega_{1}, \ldots, \omega_{p-1}, \omega_{0}, \ldots, \omega_{p-1}, \ldots\right)$ is the desired path.

If $x_{0}$ is a repelling periodic point of a rational map on $\mathbb{C}$, i.e., $r^{p}\left(x_{0}\right)=x_{0}$ and $\left|r^{p^{\prime}}\left(x_{0}\right)\right|>1$, then the cycle $\left\{x_{0}, x_{1}, \ldots, x_{p-1}\right\}$ of $x_{0}$ is repelling in the sense of Definition 6.4, because

$$
r^{p^{\prime}}\left(x_{0}\right)=r^{\prime}\left(x_{p-1}\right) r^{\prime}\left(x_{p-2}\right) \cdots r^{\prime}\left(x_{0}\right)=r^{p^{\prime}}\left(x_{k}\right),
$$

and therefore one of the inverse branches of $r^{p}$ will be contractive in the neighborhood of $x_{k}$.

If $r$ is a subshift of finite type, then every cycle is repelling, because $r$ is locally expanding.

Lemma 6.6. Suppose $x_{0}$ is a repelling fixed point. Assume that the following condition is satisfied: for every Lipschitz function $f$ on $X$, the uniform limit

$$
\lim _{n \rightarrow \infty} \frac{1}{n} \sum_{k=0}^{n-1} R_{W}^{k} f
$$

exists. Then $h_{x_{0}}$ is continuous.

Proof. We want to construct a continuous function $f$ such that $f \leq h_{x_{0}}$ and $f\left(x_{0}\right)=$ 1. Since $x_{0}$ is a repelling fixed point, there is some $\delta>0$ and $0<c<1$ such that 
for each $x$ with $d\left(x, x_{0}\right)<\delta$, there is a path $\left(z_{n}\right)_{n \geq 1}$ that starts at $x$ such that $d\left(z_{n+1}, x_{0}\right) \leq d\left(z_{n}, x\right)$ for all $n$. Then

$$
d\left(z_{n}, x_{0}\right) \leq c^{n} d\left(x, x_{0}\right), \quad n \geq 1 .
$$

In particular $\left(z_{n}\right)_{n}$ converges to $x$. So $\left(z_{n}\right)_{n}$ is in $\mathbf{N}_{x_{0}}(x)$. Therefore

$$
h_{x_{0}}(x) \geq P_{x}\left(\left\{\left(z_{n}\right)_{n}\right\}\right)=\prod_{n=1}^{\infty} W\left(z_{n}\right) .
$$

However, $W$ is Lipschitz with Lipschitz constant $L>0$, so

$$
W\left(z_{n}\right) \geq 1-L c^{n} d\left(x, x_{0}\right) .
$$

We may assume $L \delta<1 / 2$. This implies that

$$
h_{x_{0}}(x) \geq \exp \left(\sum_{n \geq 1} \log \left(1-L c^{n} d\left(x, x_{0}\right)\right)\right) .
$$

Using the inequality

$$
\log (1+a) \geq a-\frac{a^{2}}{2}, \quad a \in(-1,1),
$$

we obtain further that

$$
h_{x_{0}}(x) \geq \exp \left(-c L d\left(x, x_{0}\right) \frac{1}{1-c}-\frac{c^{2} d\left(x, x_{0}\right)^{2} L^{2}}{2\left(1-c^{2}\right)}\right)=: o(x) .
$$

The function $o(x)$ is Lipschitz, defined on a neighborhood of $x_{0}$, and its value at $x_{0}$ is 1 . Using these we can easily construct a Lipschitz function $f$ such that $f$ is smaller than $o$ and zero outside some small neighborhood of $x_{0}$, and $f\left(x_{0}\right)=1$ (e.g., take $f(x)=\eta\left(d\left(x, x_{0}\right)\right)$, where $\eta$ is some Lipschitz function on $\mathbb{R}$ with $\eta(0)=1$, $\eta(a)=0$, for $a>\delta / 2$, and $\eta$ is less than the exponential function that appeared before). Then $f \leq h_{x_{0}}$, and $f\left(x_{0}\right)=1$.

With this function, we use the hypothesis

$$
h_{f}:=\lim _{n \rightarrow \infty} \frac{1}{n} \sum_{k=0}^{n-1} R_{W}^{k} f \leq h_{x_{0}} .
$$

Also the function $h_{f}$ has to be continuous and harmonic, $R_{W} h_{f}=h_{f}$. Since $x_{0}$ is a $W$-cycle, it follows that $\left(R_{W}^{n} f\right)\left(x_{0}\right)=1$, so $h_{f}\left(x_{0}\right)=1$. But then, with Lemma 6.3. $h_{x_{0}} \leq h_{f}$. Thus $h_{x_{0}}=h_{f}$, so it is continuous.

Remark 6.7. Some comments on condition (6.9):

Given $\left(X,\left(\tau_{i}\right)_{i=1}^{N}\right)$ for some IFS, where $W$ is a Lipschitz function on $X$, we consider the following norms on $X$ :

$$
\|f\|:=\sup _{x \in X}|f(x)|, \quad\|f\|_{L}:=\nu_{W}(|f|)+\sup _{x \neq y} \frac{|f(x)-f(y)|}{d(x, y)},
$$

where $\nu_{W}$ is a probability measure to be specified below.

Assume

$$
d\left(\tau_{i} x, \tau_{i} y\right) \leq c_{i} d(x, y), \quad c:=\max _{i=1, N} c_{i}<1
$$

Also introduce

$$
v(f):=\sup _{x \neq y} \frac{|f(x)-f(y)|}{d(x, y)}
$$


and $\operatorname{Lip}(X)$ functions $f$ on $X$ such that $v(f)<\infty$. A suitable assumption on $W$ is

$$
\sum_{i=1}^{N}\left|W\left(\tau_{i} x\right)\right| \leq 1
$$

If we assume this, we get the crucial estimates which are required, so that the Cesaro convergence in (6.9) will follow from [oMa50, Lemme 4.1].

We may also pick the probability measure $\nu_{W}$ such that $\nu_{W} R_{|W|}=\nu_{|W|}$ by Markov-Kakutani Yo98.

Now

$$
\begin{aligned}
\frac{\left|R_{W} f(x)-R_{W} f(y)\right|}{d(x, y)} & \leq v(f) \sum_{i=1}^{N} c_{i}\left|W\left(\tau_{i} x\right)\right|+v(W) \sum_{i=1}^{N} c_{i}\left|f\left(\tau_{i} y\right)\right| \\
& \leq c v(f)+v(W) \sum_{i=1}^{N} c_{i}\|f\|
\end{aligned}
$$

and therefore

$$
v\left(R_{W} f\right) \leq c v(f)+\left(v(W) \sum_{i=1}^{N} c_{i}\right)\|f\| .
$$

As a result, there exists $M<\infty$ such that

$$
\begin{aligned}
\left\|R_{f}\right\|_{L}=\nu_{W}\left(\left|R_{W} f\right|\right)+v\left(R_{W} f\right) & \leq \nu_{W} R_{|W|}|f|+c v(f)+v(W) \sum_{i=1}^{N} c_{i}\|f\| \\
& \leq c\|f\|_{L}+M\|f\|,
\end{aligned}
$$

when $M$ is adjusted for the excess in the first term.

As a result, [oMa50, Lemme 4.1] applies, and (6.9) holds.

6.2. Harmonic functions associated with $W$-cycles. Let $C=\left(x_{1}, \ldots, x_{p}\right)$ be a $W$-cycle. We will extend the results in the previous section and construct continuous harmonic functions associated to cycles.

Proposition 6.8. For each $x \in X$ define the set

$$
\mathbf{N}_{C}(x):=\left\{\left(z_{n}\right)_{n \geq 1} \in \Omega_{x} \mid \lim _{n \rightarrow \infty} z_{n p}=x_{i} \text { for some } i \in\{0, \ldots, p-1\}\right\} .
$$

Define the function

$$
h_{C}(x)=P_{x}\left(\mathbf{N}_{C}(x)\right) .
$$

Then $h_{C}$ is a nonnegative, harmonic function with $h_{C}\left(x_{i}\right)=1$ for $i \in\{0, \ldots, p-1\}$. If in addition, $C$ is a repelling cycle, and for each Lipschitz function $f$ the uniform limit exists,

$$
\lim _{n \rightarrow \infty} \frac{1}{n} \sum_{k=0}^{n-1} R_{W}^{k} f=h_{f} \text { uniformly, }
$$

then $h_{C}$ is also continuous.

Proof. Note that each $x_{i}$ is a $W^{(p)}$-cycle. If $r$ is replaced by $r^{p}$, and $W^{(p)}(y)=$ $W(y) W(r(y)) \cdots W\left(r^{p-1}(y)\right)$, then $W$ becomes $W^{(p)}$. Note also that we can canonically identify the path spaces $X_{\infty}$ for $r$, and $X_{\infty}^{(p)}$ for $r^{p}$, by the bijection $\left(z_{n}\right)_{n \geq 1} \mapsto$ $\left(z_{n p}\right)_{n \geq 1}$. 
Let $\mathbf{N}_{x_{i}}(x)$ be the corresponding sets defined as in (6.4), but now working with $r^{p}$. The function

$$
g_{i}(x):=P_{x}^{(p)}\left(\mathbf{N}_{x_{i}}^{(p)}(x)\right)
$$

is nonnegative, continuous, and harmonic for $R_{W^{(p)}}=R_{W}^{p}$, as proven in Lemmas 6.2 and 6.6 .

It is clear that $(x, \omega) \mapsto \chi_{\mathbf{N}_{C}(x)}(\omega)$ is a cocycle. So, by Theorem 4.1, $h_{C}$ is harmonic and $h_{C} \leq 1$.

Note also that $\mathbf{N}_{C}(x)=\bigcup_{i=1}^{p} \mathbf{N}_{x_{i}}(x)$, a disjoint union, hence, applying $P_{x}$, $h_{C}=\sum_{i=0}^{p-1} g_{i}$, so $h_{C}$ is continuous.

Remark 6.9. Consider the case of an IFS, $\left(X, \tau_{l}\right)_{l=1}^{N}$. We want to write $\mathbf{N}_{C}(x)$ more explicitly. Clearly $\mathbf{N}_{C}(x)$ is the disjoint union of $\mathbf{N}_{x_{i}}$, where

$$
\mathbf{N}_{x_{i}}(x):=\left\{\left(z_{n}\right)_{n \geq 1} \in \Omega_{x} \mid \lim _{n \rightarrow \infty} z_{n p}=x_{i}\right\} .
$$

Take $x_{0}$ a point of a $W$-cycle of length $p$. Then, there exist $l_{0}, \ldots, l_{p-1} \in$ $\{1, \ldots, N\}$ such that

$$
\tau_{l_{p-1}} \cdots \tau_{l_{0}} x_{0}=x_{0}
$$

We make the following assumption:

$$
\tau_{\omega_{p-1}} \cdots \tau_{\omega_{0}} x_{0} \neq x_{0} \quad \text { if } \omega_{0} \ldots \omega_{p-1} \neq l_{0} \cdots l_{p-1} .
$$

We claim that

$$
\mathbf{N}_{x_{i}}(x)=\left\{\omega_{0} \cdots \omega_{k p-1} \underline{l_{0} \cdots l_{p-1}} \underline{l_{0} \cdots l_{p-1}} \cdots \mid \omega_{0}, \ldots, \omega_{k p-1} \in\{1, \ldots, N\}\right\} .
$$

Starting with (6.13) we shall use the following notation for infinite one-sided words which represent our $\mathbf{N}_{C}(x)$-cycles. (We think of these infinite words as generalized rational fractions.) After a finite number of letters, they end in an infinite repetition of a fixed finite word $w$. As indicated in (6.13), the finite word $w$ is then spelled out with an underlining, it is repeated twice, and then followed by three dots.

Take $\omega$ of the given form. Then

$$
\lim _{n \rightarrow \infty} z_{n p}=\lim _{n \rightarrow \infty}\left(\tau_{l_{p-1}} \cdots \tau_{l_{0}}\right)^{n}\left(\tau_{\omega_{k p-1}} \cdots \tau_{\omega_{0}} x\right) .
$$

But the last sequence converges to the fixed point of $\tau_{l_{p-1}} \cdots \tau_{l_{0}}$ which is $x_{0}$. This proves one of the inclusions.

For the other inclusion, take a path $\left(z_{n}\right)_{n \geq 0}$ starting at $x$ and such that $\lim _{n} z_{n p}=$ $x_{0}$. Let

$$
d:=\min \left\{d\left(\tau_{\omega_{p-1}} \cdots \tau_{\omega_{0}} x_{0}, x_{0}\right) \mid \omega_{0} \cdots \omega_{p-1} \neq l_{0} \cdots l_{p-1}\right\}
$$

There exists some $n_{0}$ such that, for $n \geq n_{0}, d\left(z_{n p}, x_{0}\right)<d / 2$.

Take such an $n$. Let $\omega_{0}, \ldots, \omega_{p-1}$ be such that $z_{(n+1) p}=\tau_{\omega_{p-1}} \cdots \tau_{\omega_{0}} z_{n p}$. We want to prove that $\omega_{0} \cdots \omega_{p-1}=l_{0} \cdots l_{p-1}$.

Suppose not. Then

$$
d\left(z_{(n+1) p}, \tau_{\omega_{p-1}} \cdots \tau_{\omega_{0}} x_{0}\right)<d\left(z_{n p}, x_{0}\right)<d / 2
$$

Also,

$$
\begin{aligned}
d \leq d\left(\tau_{\omega_{p-1}} \cdots \tau_{\omega_{0}} x_{0}, x_{0}\right) & \leq d\left(\tau_{\omega_{p-1}} \cdots \tau_{\omega_{0}} x_{0}, z_{(n+1) p}\right)+d\left(z_{(n+1) p}, x_{0}\right) \\
& <d / 2+d / 2=d,
\end{aligned}
$$


a contradiction. Therefore, as $n$ is arbitrary, the path ends in an infinite repetition of the cycle $l_{0} \cdots l_{p-1}$.

Theorem 6.10. Let $W$ be as before, and suppose it satisfies the $T Z$ condition. Suppose there exists some $W$-cycle $C$ such that $C$ intersects the closure of $O^{-}(x)$ for all $x \in X$. Assume that all $W$-cycles are repelling. In addition, assume that for every Lipschitz function $f$, the following uniform limit exists:

$$
\lim _{n \rightarrow \infty} \frac{1}{n} \sum_{k=0}^{n-1} R_{W}^{k} f
$$

Then the support of $P_{x}$ is the union $\bigcup\left\{\mathbf{N}_{C}(x) \mid C\right.$ is a $W$-cycle $\}$. Also

$$
\sum_{W \text {-cycles }} h_{C}=1 \text {. }
$$

Proof. The function $h_{C}$ is a continuous, nonnegative, harmonic function and $h_{C}(x)=P_{x}\left(\chi_{\mathbf{N}_{C}(x)}\right)$, i.e., $\chi_{\mathbf{N}_{C}(x)}$ is the corresponding cocycle. Then, using Theorem 4.1 we obtain that, for $P_{x}$-a.e., $\left(z_{n}\right)_{n \geq 1}$ outside $\mathbf{N}_{C}(x)$, and we have

$$
\lim _{n \rightarrow \infty} h_{C}\left(z_{n}\right)=\chi_{\mathbf{N}_{C}(x)}\left(\left(z_{n}\right)_{n \geq 1}\right)=0 .
$$

But we know that $h_{C}$ is continuous, and this implies that the distance from $z_{n}$ to the set of zeroes of $W$ is converging to zero. By Proposition 5.8, the zeroes of $h_{C}$ are among the $W$-cycles, because $h_{C}$ cannot be zero on some $O^{-}(x)$ as it is constant 1 on $C$. Thus for $n$ large enough, $z_{n}$ is in a small neighborhood of a point of a $W$-cycle, where the cycle is repelling. Since $z_{n+1}$ is a root of $z_{n}, z_{n+2}$ a root of $z_{n+1}$, and so on, the repelling property implies that the roots will come closer to the cycle and, in conclusion, $z_{n p}$ will converge to one of the points of the $W$-cycle. This translates into the fact that $\left(z_{n}\right)_{n \geq 1}$ is in one of the sets $\mathbf{N}_{D}(x)$, where $D$ is a $W$-cycle. In conclusion, the support of $P_{x}$ is covered by the union of these sets.

Since the sets $\mathbf{N}_{C}(x)$ are obviously disjoint and their union is $\Omega_{x}, P_{x}$-a.e., if we apply $P_{x}$ to the sum of the characteristic functions of these sets, we obtain (6.15).

\section{ITERATED FUNCTION SYSTEMS}

In this section we consider affine IFSs on $\mathbb{R}^{d}$. Let $R$ be a $d$-by- $d$ expansive matrix with coefficients in $\mathbb{R}$, i.e., its eigenvalues $\lambda$ have $|\lambda|>1$. Let $S$ be the transpose matrix $S:=R^{t}$. Let $B$ be a finite subset of $\mathbb{R}^{d}$.

Consider the following IFS on $\mathbb{R}^{d}$ :

$$
\tau_{b}(x)=R^{-1}(x+b), \quad b \in B,
$$

which we will denote by $\operatorname{IFS}(B)$.

Let $\mu_{B}$ be the invariant probability measure for the IFS $\tau_{b}(x)=R^{-1}(x+b)$, $b \in B$, i.e., the measure $\mu_{B}$ satisfies

$$
\mu=\frac{1}{N} \sum_{b \in B} \mu \circ \tau_{b}^{-1} .
$$

Lemma 7.1. Let $(B, R)$ be as above. Let $\Omega=\prod_{1}^{\infty} B$. Following Definition 3.3, define $\pi: \Omega \rightarrow X_{B}$, by $\pi(b)=\sum_{k=1}^{\infty} R^{-k} b_{k}$. Let $N=\# B$, and let $\nu_{N}$ be the Bernoulli measure $\left(\frac{1}{N}, \ldots, \frac{1}{N}\right)$ on $\Omega$. Then $\mu$ in (7.2) is $\mu=\nu_{N} \circ \pi^{-1}$. 
Proof. Follows from the definitions.

Define

$$
m_{B}(x):=\frac{1}{\sqrt{N}} \sum_{b \in B} e^{2 \pi i b \cdot x}, \quad x \in \mathbb{R}^{d} .
$$

We denote by $\hat{\mu}_{B}$ its Fourier transform

$$
\hat{\mu}_{B}(t)=\int_{X} e^{2 \pi i t \cdot x} d \mu_{B}(x), \quad t \in \mathbb{R}^{d} .
$$

Then one checks that

$$
\hat{\mu}_{B}(t)=\frac{m_{B}\left(S^{-1} t\right)}{\sqrt{N}} \hat{\mu}_{B}\left(S^{-1} t\right), \quad t \in \mathbb{R}^{d} .
$$

Definition 7.2. We call a pair of subsets $\{A, B\}$ a Hadamard pair, if $\# A=\# B=$ : $N$ and the matrix

$$
\frac{1}{\sqrt{N}}\left(e^{2 \pi i a \cdot b}\right)_{a \in A, b \in B} \text { is unitary. }
$$

We will further assume that $(B, L, R)$ is in Hadamard duality (see Definition 3.6), i.e., that there exists $L$ such that $\left\{R^{-1} B, L\right\}$ form a Hadamard pair.

Associated to $L$, we have the iterated function system $\operatorname{IFS}(L)$, defined by the maps

$$
\tau_{l}(x)=S^{-1}(x+l), \quad l \in L .
$$

We denote by $X_{L}$, the attractor of the IFS $\left(\tau_{l}\right)_{l \in L}$.

In the following, we will use our theory on the iterated function system $\operatorname{IFS}(L)$, so the Ruelle operator is associated to $L$. The first result is that if

$$
W_{B}:=\frac{1}{N}\left|m_{B}\right|^{2},
$$

then $W_{B}$ satisfies the condition (6.1):

Proposition 7.3. The function $W_{B}$ satisfies the following condition:

$$
R_{W_{B}} 1=1 .
$$

Also, $\{0\}$ is an $m_{B}$-cycle.

Proof. We have to prove that

$$
\sum_{l \in L}\left|m_{B}\left(S^{-1}(x+l)\right)\right|^{2}=N, \quad x \in \mathbb{R}^{d} .
$$

Note that the column vector $\left(v^{t}\right.$ denotes the matrix transpose of $\left.v\right)$

$$
\begin{aligned}
m_{B}\left(S^{-1}(x+l)\right)_{l \in L}^{t} & =\left(\frac{1}{\sqrt{N}} \sum_{b \in B} e^{2 \pi i b \cdot S^{-1}(x+l)}\right)_{l \in L}^{t} \\
& =\frac{1}{\sqrt{N}}\left(e^{2 \pi i b \cdot S^{-1} l}\right)_{b \in B, l \in L} \cdot\left(e^{2 \pi i b \cdot S^{-1} x}\right)_{b \in B}^{t} .
\end{aligned}
$$

But the matrix is unitary, so it preserves norms, and the norm of the vector $\left(e^{2 \pi i b \cdot S^{-1} x}\right)_{b \in B}^{t}$ is $\sqrt{N}$. This implies (7.5). 
Since $W_{B}$ satisfies $R_{W_{B}} 1=1$, we can construct $P_{x}$ from it (see (4.4)) and use the entire theory developed in the previous sections.

Since $R$ is expansive, for $a$ large enough, all maps $\tau_{i}$ map the closed ball $B(0, a)$ into itself. Indeed, $\left\|S^{-1}\right\|<1$ and let $M:=\max \left\|b_{i}\right\|$. Then, if

$$
a>\left\|S^{-1}\right\| M /\left(1-\left\|S^{-1}\right\|\right),
$$

then

$$
\left\|S^{-1}(x+b)\right\| \leq\left\|S^{-1}\right\|(a+M) \leq a .
$$

Therefore, we can consider the ground space to be the closed ball $B(0, a)$ and we can therefore construct $P_{x}$ for any $x$ in this ball. Note also that this does not depend on the choice of $a$, therefore we can define $P_{x}$ for all $x \in \mathbb{R}^{d}$.

\section{Spectrum of a fractal measure}

As in [JoPe96], we make the following assumptions:

$$
\begin{gathered}
\left\{R^{-1} B, L\right\} \text { form a Hadamard pair, } \# B=\# L=: N, \\
R^{n} b \cdot l \in \mathbb{Z}, \text { for } b \in B, l \in L, n \geq 0, \\
\qquad 0 \in B, 0 \in L .
\end{gathered}
$$

Here $S=R^{t}$ is the transpose of the matrix $R$ in (7.1).

8.1. Fixed points. Now suppose that $l_{0} \in L$ gives a $W_{B}$-cycle, i.e., the fixed point $x_{l_{0}} \in X_{L}$ of the map $\tau_{l_{0}}$ has the property that $W_{B}\left(x_{l_{0}}\right)=1$.

Proposition 8.1. If $x_{l_{0}}$ is a $W_{B}$-cycle, then, for $\omega_{0}, \ldots, \omega_{n} \in L$, set

$$
k_{l_{0}}(\omega):=\omega_{0}+S \omega_{1}+\cdots+S^{n} \omega_{n}-S^{n+1}(S-I)^{-1} l_{0} .
$$

Then, for all $x \in \mathbb{R}^{d}$,

$$
P_{x}\left(\left\{\left(\omega_{0} \cdots \omega_{n} l_{0} l_{0} \cdots\right)\right\}\right)=\left|\hat{\mu}_{B}\left(x+k_{l_{0}}(\omega)\right)\right|^{2} .
$$

Proof. Since $x_{l_{0}}$ is the fixed point of $\tau_{l_{0}}$, we have $S^{-1}\left(x_{l_{0}}+l_{0}\right)=x_{l_{0}}$, so $x_{l_{0}}=$ $(S-I)^{-1} l_{0}$. Since this is a $W_{B}$-cycle, it follows that

$$
\left|\sum_{b \in B} e^{2 \pi i b \cdot x_{l_{0}}}\right|=N .
$$

However, there are $N$ terms in the sum, one of them is 1 , and all have absolute value 1 . This implies that we have equality in the triangle inequality applied to this situation, so $e^{2 \pi i b \cdot x_{0}}=1$ for all $b \in B$. Therefore we see that $b \cdot(S-I)^{-1} l_{0} \in \mathbb{Z}$, for all $b \in B$, and

$$
m_{B}\left(x+(S-I)^{-1} l_{0}\right)=m_{B}(x), \quad x \in \mathbb{R}^{d} .
$$

Also for $n \geq 0, b \in B$, we have

$$
\begin{aligned}
b \cdot S^{n+1}(S-I)^{-1} l_{0} & =b \cdot\left(\left(S^{n+1}-I\right)(S-I)^{-1} l_{0}+(S-I)^{-1} l_{0}\right) \\
& =b \cdot\left(\left(I+S+\cdots+S^{n}\right) l_{0}+(S-I)^{-1} l_{0}\right) \in \mathbb{Z},
\end{aligned}
$$

so

$$
m_{B}\left(x+S^{n+1}(S-I)^{-1} l_{0}\right)=m_{B}(x), \quad x \in \mathbb{R}^{d}, b \in B .
$$


Let $\omega_{0}, \ldots, \omega_{n} \in L, j \geq 0$. We have, with $k_{0}(\omega):=\omega_{0}+S \omega_{1}+\cdots+S^{n} \omega_{n}$ and $k_{l_{0}}(\omega)=k_{0}(\omega)-S^{n+1}(S-I)^{-1} l_{0}$, the formula

$$
\begin{aligned}
m_{B}\left(\tau_{l_{0}}^{j} \tau_{\omega_{n}} \cdots \tau_{\omega_{0}} x\right)= & m_{0}\left(S ^ { - ( n + j + 1 ) } \left(x+\omega_{0}+S \omega_{1}+\cdots+S^{n} \omega_{n}\right.\right. \\
& \left.\left.+S^{n+1} l_{0}+\cdots+S^{n+j} l_{0}\right)\right) \\
= & m_{B}\left(S^{-(n+j+1)}\left(x+k_{0}(\omega)+S^{n+1}\left(I+S+\cdots+S^{j-1}\right) l_{0}\right)\right) \\
= & m_{B}\left(S^{-(n+j+1)}\left(x+k_{0}(\omega)+S^{n+1}\left(S^{j}-I\right)(S-I)^{-1} l_{0}\right)\right) \\
= & m_{B}\left(S^{-(n+j+1)}\left(x+k_{0}(\omega)-S^{n+1}(S-I)^{-1} l_{0}\right)-l_{0}\right) \\
= & m_{B}\left(S^{-(n+j+1)}\left(x+k_{0}(\omega)-S^{n+1}(S-I)^{-1} l_{0}\right)\right) \\
= & m_{B}\left(S^{-(n+j+1)}\left(x+k_{l_{0}}(\omega)\right) .\right.
\end{aligned}
$$

Also, using the $\mathbb{Z}^{d}$-periodicity of $m_{B}$ and (8.5), for $i \leq n$, we get

$$
\begin{aligned}
m_{B}\left(\tau_{\omega_{i}} \cdots \tau_{\omega_{0}} x\right)= & m_{B}\left(S^{-(i+1)}\left(x+\omega_{0}+S \omega_{1}+\cdots S^{i} \omega_{i}\right)\right) \\
= & m_{B}\left(S ^ { - ( i + 1 ) } \left(x+\omega_{0}+S \omega_{1}+\cdots+S^{i} \omega_{i}\right.\right. \\
& \left.\left.\quad+S^{i+1} \omega_{i+1}+\cdots+S^{n} \omega_{n}-S^{n+1}(S-I)^{-1} l_{0}\right)\right) \\
= & m_{B}\left(S^{-(i+1)}\left(x+k_{l_{0}}(\omega)\right)\right) .
\end{aligned}
$$

With these relations, Lemma 6.1, and relation (17.3), we can conclude that

$$
\begin{aligned}
P_{x}\left(\left\{\left(\omega_{0} \cdots \omega_{n} l_{0} l_{0} \cdots\right)\right\}\right) & =\prod_{j=1}^{\infty} \frac{\left|m_{B}\left(S^{-j}\left(x+k_{l_{0}}(\omega)\right)\right)\right|^{2}}{N} \\
& =\left|\hat{\mu}_{B}\left(x+k_{l_{0}}(\omega)\right)\right|^{2} .
\end{aligned}
$$

8.2. From fixed points to longer cycles. We now analyze how the elements are changing when passing from scale $R$ to $R^{p}$. If

$$
B^{(p)}:=\left\{b_{0}+R b_{1}+\cdots+R^{p-1} b_{p-1} \mid b_{0}, \ldots, b_{p-1} \in B\right\}
$$

and

$$
L^{(p)}:=\left\{l_{0}+S l_{1}+\cdots+S^{p-1} l_{p-1} \mid l_{0}, \ldots, l_{p-1} \in L\right\},
$$

then the triple $\left(B^{(p)}, L^{p}, R^{p}\right)$ satisfies the conditions mentioned above. The fact that they form a Hadamard pair follows from the fact that $R_{W_{B}(p)} 1=1$ and LaWa02, Lemma 2.1]. See also [JoPe96] and Example 3.5 above. Specifically, if $U$ is the Hadamard matrix of $(B, L)$, then $U \otimes \cdots \otimes U$ is the Hadamard matrix of $\left(B^{(p)}, L^{(p)}\right)$.

Lemma 8.2. Let $(B, L, R)$ be a Hadamard system, and let $p \in \mathbb{N}$. Let $m_{B^{(p)}}$ and $P_{x}^{(p)}$ be constructed from $B^{(p)}$. Then we have

$$
m_{B}\left(\tau_{\omega_{0}} x\right) \cdots m_{B}\left(\tau_{\omega_{p-1}} \cdots \tau_{\omega_{0}} x\right)=m_{B^{(p)}}\left(\tau_{\omega_{p-1}} \cdots \tau_{\omega_{0}} x\right)
$$

and

$$
P_{x}(\{\omega\})=P_{x}^{(p)}(\{\omega\}), \quad \text { for all } \omega \in \Omega \text {. }
$$


Proof. Note that

$$
\begin{aligned}
m_{B^{(p)}}(x) & =\frac{1}{\sqrt{N^{p}}} \sum_{b_{0}, \ldots, b_{p-1} \in B} e^{2 \pi i\left(b_{0}+R b_{1}+\cdots+R^{p-1} b_{p}\right) \cdot x} \\
& =m_{B}(x) m_{B}(S x) \cdots m_{B}\left(S^{p-1} x\right)=m_{B}^{(p)}(x) .
\end{aligned}
$$

The iterated function system $\operatorname{IF} S\left(B^{(p)}\right)$ has the same attractor $X_{B}$ as $\operatorname{IFS}(B)$. The same is true for $L$. Thus

$$
\hat{\mu}_{B}=\hat{\mu}_{B(p)} .
$$

There is a canonical identification between $\Omega=L^{\mathbb{N}}$ and $\Omega^{(p)}=\left(L^{(p)}\right)^{\mathbb{N}}$ given by

$$
\left(\omega_{0} \omega_{1} \cdots\right) \leftrightarrow\left(\left(\omega_{0} \cdots \omega_{p-1}\right)\left(\omega_{p} \cdots \omega_{2 p-1}\right) \cdots\right) .
$$

Also note that for $\omega_{i} \in L$,

$$
\begin{aligned}
& m_{B}\left(\tau_{\omega_{0}} x\right) \cdots m_{B}\left(\tau_{\omega_{p-1}} \cdots \tau_{\omega_{0}} x\right) \\
& \quad=m_{B}\left(S^{-1}\left(x+\omega_{0}\right)\right) \\
& \quad \cdot m_{B}\left(S^{-2}\left(x+\omega_{0}+S \omega_{1}\right)\right) \cdots m_{B}\left(S^{-p}\left(x+\omega_{0}+\cdots+S^{p-1} \omega_{p-1}\right)\right) \\
& \quad=m_{B}\left(S^{-1}\left(x+\omega_{0}+\cdots+S^{p-1} \omega_{p-1}\right)\right) \cdots m_{B}\left(S^{-p}\left(x+\omega_{0}+\cdots+S^{p-1} \omega_{p-1}\right)\right) \\
& =m_{B}\left(\tau_{\omega_{p-1}} \cdots \tau_{\omega_{0}} x\right),
\end{aligned}
$$

where we used periodicity in the second equality.

Then, for $x \in \mathbb{R}^{d}$, we have

$$
\begin{aligned}
P_{x}\left(\left\{\omega_{0} \cdots \omega_{n} \cdots\right\}\right) & =\prod_{j=1}^{\infty} \frac{\left|m_{B}\left(\tau_{\omega_{k}} \cdots \tau_{\omega_{0}} x\right)\right|^{2}}{N} \\
& =\prod_{j=1}^{\infty} \frac{\left|m_{B^{(p)}}\left(\tau_{\omega_{k p-1}} \cdots \tau_{\omega_{0}} x\right)\right|^{2}}{N^{p}}=P_{x}^{(p)}\left(\left\{\omega_{0} \cdots \omega_{n} \cdots\right\}\right) .
\end{aligned}
$$

8.3. Cycles. Assume now $W_{B}$ has a cycle of length $p: C:=l_{0} \cdots l_{p-1}$. This means that for the fixed point $x_{C}$ of $\tau_{l_{p-1}} \cdots \tau_{l_{0}}$, the following relations hold:

$$
W_{B}\left(\tau_{l_{k}} \cdots \tau_{l_{0}} x_{C}\right)=1, \quad k \in\{0, \ldots, p-1\} .
$$

Proposition 8.3. Suppose $C=l_{0} \cdots l_{p-1}$ is a $W_{B}$-cycle. For $\omega_{0}, \ldots, \omega_{k p-1} \in L$, denote by

$$
k_{l_{0} \cdots l_{p-1}}(\omega):=\omega_{0}+S \omega_{1}+\cdots+S^{k p-1} \omega_{k p-1}-S^{k p}\left(S^{p}-I\right)^{-1}\left(l_{0}+S l_{1}+\cdots+S^{p-1} l_{p-1}\right) .
$$

Then

$$
P_{x}\left(\left\{\omega_{0} \cdots \omega_{k p-1} \underline{l_{0} \cdots l_{p-1}} \underline{l_{0} \cdots l_{p-1}} \cdots\right\}\right)=\left|\hat{\mu}_{B}\left(x+k_{l_{0} \cdots l_{p-1}}(\omega)\right)\right|^{2} .
$$

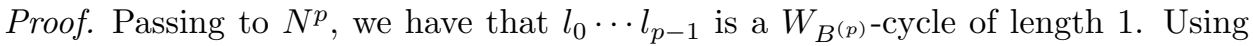
the previous analysis, we obtain that

$$
\begin{aligned}
& P_{x}\left(\left\{\omega_{0} \cdots \omega_{k p-1} l_{0} \cdots l_{p-1} l_{0} \cdots l_{p-1} \cdots\right\}\right) \\
& =P_{x}^{(p)}\left(\left\{\omega_{0} \cdots \omega_{k p-1} \underline{l_{0} \cdots l_{p-1}} l_{0} \cdots l_{p-1} \cdots\right\}\right) \\
& =\left|\hat{\mu}_{B(p)}\left(x+k_{l_{0} \cdots l_{p-1}}(\omega)\right)\right|^{2}=\left|\hat{\mu}_{B}\left(x+k_{l_{0} \cdots l_{p-1}}(\omega)\right)\right|^{2} \text {. }
\end{aligned}
$$


8.4. Spectrum and cycles. We are now able to compute the spectrum of the fractal measure $\mu_{B}$.

Theorem 8.4. Suppose conditions (8.1)-(8.3) are satisfied, and that $W_{B}$ satisfies the $T Z$ condition in Definition [5.6. Let $\Lambda \subset \mathbb{R}^{d}$ be the smallest set that contains $-C$ for all $W_{B}$-cycles $C$, and such that $S \Lambda+L \subset \Lambda$. Then

$$
\left\{e^{2 \pi i \lambda \cdot x} \mid \lambda \in \Lambda\right\}
$$

is an orthonormal basis for $L^{2}\left(\mu_{B}\right)$.

Proof. We verify the hypotheses of Theorem 6.10. First note that 0 is a $W_{B}$-cycle, and for any $x \in \mathbb{R}^{d}$,

$$
\lim _{n \rightarrow \infty} \underbrace{\tau_{0} \cdots \tau_{0}}_{n \text { times }} x=0
$$

so 0 belongs to the closure of the inverse orbit of any point.

From Remark 6.5, we see that all $W_{B}$-cycles are repelling.

The uniform convergence of the Cesaro sums in (6.14) follow from Remark 6.7

Hence, with Theorem 6.10 we can conclude that

$$
\sum_{C \text { is a } W_{B} \text {-cycle }} h_{C}(x)=1, \quad x \in \mathbb{R}^{d} .
$$

We will write this sum in terms of $\hat{\mu}_{B}$.

We use Remark 6.9, and we check that if $x_{0}$ is the fixed point of $\tau_{l_{p-1}} \cdots \tau_{l_{0}}$, then $x_{0}$ is not fixed by any other $\tau_{\omega_{p-1}} \cdots \tau_{\omega_{0}}$.

But we have that $R_{W_{B}} 1=1$ so $R_{W_{B}(p)} 1=1$, and this rewrites as

$$
\sum_{\omega_{0}, \ldots, \omega_{p-1}} W_{B}\left(S^{-p}\left(x+\omega_{0}+\cdots+S^{p-1} \omega_{p-1}\right)\right)=1
$$

If one takes $x=l_{0}+\cdots+S^{p-1} l_{p-1}$, then one of the terms in the sum is 1 , so the others have to be zero which implies that $\omega_{0}+\cdots+S^{p-1} \omega_{p-1} \neq l_{0}+\cdots+S^{p-1} l_{p-1}$ if $\omega_{0} \cdots \omega_{p-1} \neq l_{0} \cdots l_{p-1}$. Therefore, a simple calculation shows that the maps $\tau_{\omega_{p-1}} \cdots \tau_{\omega_{0}}$ and $\tau_{l_{p-1}} \cdots \tau_{l_{0}}$ will have different fixed points.

We can use now Remark 6.9 to see that the paths in $\mathbf{N}_{C}$ are of the form $\omega_{0} \cdots \omega_{k p-1} l_{0} \cdots l_{p-1} \underline{l_{0} \cdots l_{p-1}} \cdots$, where $l_{0} \cdots l_{p-1}$ give the points of the $W_{B^{-}}$ cycle. We will use the simpler notation $k(\omega):=k_{l_{0} \cdots l_{p-1}}\left(\omega_{0} \cdots \omega_{k p-1}\right)$.

We will show that

$$
\begin{aligned}
& \Lambda=\left\{k_{l_{0} \cdots l_{p-1}}(\omega) \mid l_{0} \cdots l_{p-1} \text { is a point in a } W_{B^{-c y c l e}},\right. \\
& \left.\omega=\omega_{0} \cdots \omega_{n p-1} \in L^{n p}, n \geq 0\right\},
\end{aligned}
$$

but first we prove that the set of frequencies given in the right side of this equality will yield an ONB.

We have, with Proposition 8.3 ,

$$
1=\sum_{C} \sum_{\omega \in \mathbf{N}_{C}} P_{x}(\{\omega\})=\sum_{C} \sum_{\omega \in \mathbf{N}_{C}}\left|\hat{\mu}_{B}(x+k(\omega))\right|^{2}, \quad x \in \mathbb{R}^{d} .
$$

Take $x=-k(\omega)$ for some $\omega$ in one of the sets $\mathbf{N}_{C}$. Then, since $\hat{\mu}_{B}(0)=1$ it follows that

$$
\hat{\mu}_{B}\left(-k(\omega)+k\left(\omega^{\prime}\right)\right)=0
$$

for all $\omega^{\prime} \neq \omega$. In particular $k(\omega) \neq k\left(\omega^{\prime}\right)$ for $\omega \neq \omega^{\prime}$, and

$$
e_{k(\omega)} \perp e_{k\left(\omega^{\prime}\right)} \text {. }
$$


Also, we can rewrite (8.8) as

$$
\left\|e_{-x}\right\|^{2}=\sum_{C} \sum_{\omega \in \mathbf{N}_{C}}\left|\left\langle e_{-x} \mid e_{k(\omega)}\right\rangle\right|^{2} .
$$

But, since the functions $e_{k(\omega)}$ are mutually orthogonal, this implies that $e_{-x}$ belongs to the closed linear span of $\left(e_{k(\omega)}\right)_{\omega}$. The Stone-Weierstrass theorem implies that the linear span of $\left(e_{-x}\right)_{x \in \mathbb{R}^{d}}$ is dense in $C\left(X_{B}\right)$. In conclusion, the functions $e_{k(\omega)}$ span $L^{2}\left(\mu_{B}\right)$, and they are orthogonal so they form an orthonormal basis for $L^{2}\left(\mu_{B}\right)$.

It remains to check (8.7). We denote by $\Lambda^{\prime}$ the right side of (8.7). Some simple computations are sufficient to prove the following: if $x_{l_{0} \cdots l_{p-1}}$ is the fixed point for $\tau_{l_{p-1}} \cdots \tau_{l_{0}}$, then

$$
x_{l_{0} \cdots l_{p-1}}=\left(S^{p}-I\right)^{-1}\left(l_{0}+\cdots+S^{p-1} l_{0}\right), \quad S x_{l_{0} \cdots l_{p-1}}=x_{l_{p-1} l_{0} \cdots l_{p-2}}+l_{p} .
$$

For $\omega_{0}, \ldots, \omega_{k p-1} \in L$,

$$
k_{l_{0} \cdots l_{p-1}}\left(\omega_{0} \cdots \omega_{k p-1}\right)=k_{l_{0} \cdots l_{p-1}}\left(\omega_{0} \cdots \omega_{k p-1} l_{0} \cdots l_{p-1}\right) .
$$

Also

$$
\begin{gathered}
k_{l_{0} \cdots l_{p-1}}\left(\omega_{0} \cdots \omega_{k p-1}\right)=S k_{l_{1} \cdots l_{p-1} l_{0}}\left(\omega_{1} \cdots \omega_{k p-1} l_{0}\right)+\omega_{0} \\
k_{l_{0} \cdots l_{p-1}}(\emptyset)=-x_{l_{0} \cdots l_{p-1}}, \quad \text { where } \emptyset \text { is the empty word. }
\end{gathered}
$$

With these, one obtains that

$$
\begin{aligned}
S k_{l_{0} \cdots l_{p-1}}\left(\omega_{0} \cdots \omega_{k p-1}\right)+\omega_{-1} & =S k_{l_{0} \cdots l_{p-1}}\left(\omega_{0} \cdots \omega_{k p-1} l_{0} \cdots l_{p-1}\right)+\omega_{-1} \\
& =k_{l_{p-1} l_{0} \cdots l_{p-2}}\left(\omega_{0} \cdots \omega_{k p-1} l_{0} \cdots l_{p-2}\right) .
\end{aligned}
$$

This shows that $S \Lambda^{\prime}+L \subset \Lambda^{\prime}$.

On the other hand, successive applications of (8.9) show that every point in $\Lambda^{\prime}$ can be obtained from one of the points $-x_{l_{0} \cdots l_{p-1}}$ after several applications of operations of the form $x \mapsto S x+l$. This implies that $\Lambda^{\prime}$ has the minimality property of $\Lambda$, so $\Lambda^{\prime}=\Lambda$.

Remark 8.5. Consider a system $(X, \mu)$ with $X$ a compact subset of $\mathbb{R}^{d}$. Following Definition 2.1. we say that a subset $\Lambda$ of $\mathbb{R}^{d}$ is a Fourier basis set if $\left\{e_{\lambda} \mid \lambda \in \Lambda\right\}$ is an orthogonal basis in $L^{2}(X, \mu)$. These $\Lambda$ sets were introduced in [JoPe98, and [JoPe99]. They are motivated by [Fu74, and are of interest even for concrete simple examples: if $X$ is the $d$-cube in $\mathbb{R}^{d}$, and $\mu$ is the Lebesgue measure, all the Fourier basis sets $\Lambda$ were found in [JoPe99]. (See also LaSh94, [LRW00, LaWa00, JoPe93, and [oPe98.)

If $\left(X_{B}, \mu_{B}\right)$ is the IFS system constructed from $\tau_{0}(x)=x / 4, \tau_{2}(x)=(x+2) / 4$, i.e., $B=\{0,2\}, R=4$, then we showed in [JoPe98 that $\left(X_{B}, \mu_{B}\right)$ has Fourier basis sets. We recalled one of them in Section 2 above. Even though this last system is one of the simplest fractals (e.g., with Hausdorff dimension = scaling dimension $=\frac{1}{2}$ ), all its Fourier basis sets $\Lambda$ are not known. Here we list some of them which arise as consequences of our duality analysis from the study of pairs $(B, L)$ with the Hadamard property.

Each set $L=\left\{0, l_{1}\right\}$, where $l_{1}$ is an odd integer, gives rise to an ONB set $\Lambda\left(l_{1}\right)$ as in Theorem 8.4. The case $\Lambda(1)$ was included in [JoPe98,

$$
\Lambda(1)=\{0,1,4,5,16,17,20,21,24,25, \ldots\} .
$$


The only $W_{B}$-cycle which contributes to $\Lambda(1)$ is the one cycle $\{0\}$. Since $L=$ $\left\{0, l_{1}\right\}$, the periodic points in $X_{L}$ which generate cycles are $000 \cdots$ and $l_{1} l_{1} l_{1} \ldots$ for the one-cycles. There can be only one two-cycle, i.e., the one generated by $\left(0, l_{1}\right)$. The two three-cycles are generated by $\left(0 l_{1} l_{1}\right)$, and $\left(l_{1} 00\right)$, respectively. The first $\Lambda\left(l_{1}\right)$ with two one-cycles which are also $W_{B}$-cycles is $\Lambda(3)$. The first $\Lambda\left(l_{1}\right)$ with a $W_{B^{-}}$-two-cycle is $\Lambda(15)$, and the two-cycle is $\{1,4\}$. The first $W_{B^{-}}$ three-cycle occurs in $\Lambda(63)$, and it is $\{16,4,1\}$. We listed $\Lambda(1)$, and the next is $\Lambda(3)=\left\{\omega_{0}+4 \omega_{1}+\cdots+4^{n} \omega_{n} \mid \omega_{i} \in\{0,3\}, n=0,1, \ldots\right\} \cup\left\{\omega_{0}+4 \omega_{1}+\cdots+\right.$ $\left.4^{n} \omega_{n}-1 \mid \omega_{i} \in\{0,-3\}, n=0,1, \ldots\right\}$. If $l_{1} \in\{5,7,9,11,13,17,19,23,29\}$, then $\Lambda\left(l_{1}\right)=l_{1} \Lambda(1)=\left\{l_{1} \lambda \mid \lambda \in \Lambda(1)\right\}$; but $\Lambda(3), \Lambda(15), \Lambda(27)$, and $\Lambda(63)$ are more subtle. Nonetheless, they can be computed with the aid of Theorem 8.4 ,

At the conclusion of this paper we received a preprint Str05] which proves a striking convergence theorem for the $\Lambda$-Fourier series defined on $(B, L)$ systems $\left(X_{B}, \mu_{B}\right)$; i.e., convergence of $\sum_{\lambda \in \Lambda} c_{\lambda} e_{\lambda}$ for functions in $C\left(X_{B}\right)$.

\section{The case of Lebesgue measure}

Our main results from Sections 78 have been focused on the fractal case; i.e., on the harmonic analysis $L^{2}(\mu)$ for IFS-measures $\mu$ with compact support $X$ in $\mathbb{R}^{d}$, and with $(X, \mu)$ having a Hausdorff dimension (= similarity dimension) which is smaller than $d$. More generally, when the transformations $\left(\tau_{i} ; i=1, \ldots, N\right)$ in some contractive IFS are given, the measure $\mu$ is determined up to scale by the equation

$$
\mu=\frac{1}{N} \sum_{i=1}^{N} \mu \circ \tau_{i}^{-1},
$$

as is well known from Hut81.

We now outline a class of IFSs where the maps $\left(\tau_{i}\right)$ act on a compact subset $X$ in $\mathbb{R}^{d}$, and where (9.1) is satisfied by the $d$-dimensional Lebesgue measure $\lambda$, restricted to $X$. In Section 7 , for the affine Hadamard case, we studied Hadamard systems $(B, L, R)$, with the two subsets $B$ and $L$ chosen such that the number $\#(B)=\#(L)=: N$ is strictly smaller than $|\operatorname{det}(R)|$. Then the self-similar measure $\mu$ of (9.1) will have fractal dimension.

However, in this section we will specialize further to the case when $N=|\operatorname{det}(R)|$ holds, and when the vectors in the set $B$ are chosen to be in a one-to-one correspondence with the elements in the finite quotient group $\mathbb{Z}^{d} / R\left(\mathbb{Z}^{d}\right)$. This choice, and Lemma 7.1. imply that $\mu$ in (9.1) is a multiple of the $d$-Lebesgue measure. Similarly, the set $L$ in the pairing is chosen to be in one-to-one correspondence with $\mathbb{Z}^{d} / S\left(\mathbb{Z}^{d}\right)$, where $S$ is the transpose of $R$. These special systems $(B, L, R, X, \mu)$ have a certain rigidity, they have connection to wavelet theory, and they have been studied earlier in [JoPe96], LaWa97, and [BrJo99. It turns out that the resulting measure $\mu$ from (9.1) will then be an integral multiple of the standard $d$-dimensional Lebesgue measure, restricted to $X$. In fact, the Lebesgue measure of $X, \lambda(X)$ will be an integer $1,2, \ldots$ (The case $\lambda(X)=1$ is a $d$-dimensional Haar wavelet.)

Further, the support sets $X$ will tile $\mathbb{R}^{d}$ with translations from a certain lattice $\Gamma$ in $\mathbb{R}^{d}$ such that the order of the group $\mathbb{Z}^{d} / \Gamma$ equals $\lambda(X)$. This tiling property is defined relative to Lebesgue measure, i.e., the requirement that distinct $\Gamma$ translates of $X$ overlap on sets of at most zero Lebesgue measure in $\mathbb{R}^{d}$. While $X$ will automatically have a nonempty interior, it typically has a fractal boundary; see LaWa97] and [JoPe96]. 
The main point below is the presentation of an example in $\mathbb{R}^{2}$ where the $P_{x^{-}}$ measure of the union of the sets $\mathbf{N}_{C}$, as $C$ ranges over the $W$-cycles, is strictly less than 1. (For the measures of $P_{x}$, see Lemma 6.2). This means that the dimension of the null-space $N_{C(X)}\left(I-R_{W}\right)$ is strictly larger than the number of $W$-cycles. Moreover, in view of Theorem 6.10, our condition TZ (Definition [5.6) for $W$ will not be satisfied in this example, and we sketch the geometric significance of this fact.

Example 9.1. In this example we give a system $(B, L, R)$, for which the TZ condition of Definition 5.6 fails to hold. Yet the Hilbert space $L^{2}\left(X_{B}\right)$ has an orthonormal basis of Fourier frequencies $e_{\lambda}$ indexed by $\lambda \in \Lambda$ in a certain lattice. We further compute the part of this orthonormal basis which is generated by the $W_{B}$-cycles.

Specifications $d=2$ :

$$
\begin{gathered}
B=\left\{\left(\begin{array}{l}
0 \\
0
\end{array}\right),\left(\begin{array}{l}
3 \\
0
\end{array}\right),\left(\begin{array}{l}
0 \\
1
\end{array}\right),\left(\begin{array}{l}
3 \\
1
\end{array}\right)\right\}, \\
L=\left\{\left(\begin{array}{l}
0 \\
0
\end{array}\right),\left(\begin{array}{l}
1 \\
0
\end{array}\right),\left(\begin{array}{l}
0 \\
1
\end{array}\right),\left(\begin{array}{l}
1 \\
1
\end{array}\right)\right\}, \\
R=\left(\begin{array}{ll}
2 & 1 \\
0 & 2
\end{array}\right) .
\end{gathered}
$$

We shall use both the IFSs coming from $B$ and from $L$, i.e.,

$$
\begin{gathered}
\tau_{b}(x)=R^{-1}(x+b), \quad b \in B, \\
\tau_{l}(x)=S^{-1}(x+l), \quad l \in L, \quad S=R^{t} .
\end{gathered}
$$

The corresponding compact sets in $\mathbb{R}^{2}$ will be denoted $X_{B}$ and $X_{L}$.

The reader may check that the pair $\left(R^{-1} B, L\right)$ satisfies the Hadamard condition for the $4 \times 4$ Hadamard matrix in (3.3) corresponding to $u=i=\sqrt{-1}$. Moreover the invariant measures corresponding to both of the systems $\left(\tau_{b}\right)$ and $\left(\tau_{l}\right)$ in (9.1) are multiples of the 2-dimensional Lebesgue measure $\lambda$. Specifically $\lambda\left(X_{B}\right)=3$, and $X_{B}$ tiles $\mathbb{R}^{2}$ under translations by the lattice $\Gamma=3 \mathbb{Z} \times \mathbb{Z}$, i.e.,

$$
\begin{gathered}
\mathbb{R}^{2}=\bigcup_{\gamma \in \Gamma}\left(X_{B}+\gamma\right) \text { and } \\
\lambda\left(\left(X_{B}+\gamma\right) \cap\left(X_{B}+\gamma^{\prime}\right)\right)=0, \quad \gamma \neq \gamma^{\prime} .
\end{gathered}
$$

From this, LaWa97, and the theory of Fourier series, it follows that the dual lattice

$$
\Gamma^{0}=\left(\frac{1}{3} \mathbb{Z}\right) \times \mathbb{Z}
$$

defines an orthogonal basis in $L^{2}\left(X_{B}\right)$, i.e., that the functions $e_{\lambda}(x)=e^{i 2 \pi \lambda \cdot x}$, $\lambda \in \Gamma^{0}$, form an orthogonal basis for $L^{2}\left(X_{B}\right)$.

We now turn to the $W_{B}$-cycles for the other IFS, i.e., for $\left(X_{L},\left(\tau_{l}\right)\right)$. The function $m_{B}$ is

$$
m_{B}(x, y)=\frac{1}{2}\left(1+e^{2 \pi i 3 x}+e^{2 \pi i y}+e^{2 \pi i(3 x+y)}\right) .
$$

Then $W_{B}(x, y)=1$ if and only if $3 x \in \mathbb{Z}$ and $y \in \mathbb{Z}$.

It follows from the discussion in Section 8 that if $x \in \mathbb{R}^{2}$ is a point in a $p$-cycle, it must have the form

$$
x=\left(S^{p}-I\right)^{-1}\left(S^{p-1} l_{0}+\cdots+l_{p-1}\right),
$$


where $l_{i} \in L$. If the $p$-cycle is also a $W_{B}$-cycle, then $x \in X_{L} \cap\left\{x \mid W_{B}(x)=1\right\}$. We check that this is satisfied if $p=1$, and we get the 4 one-cycles

$$
\left\{\left(\begin{array}{l}
0 \\
0
\end{array}\right)\right\},\left\{\left(\begin{array}{c}
1 \\
-1
\end{array}\right)\right\},\left\{\left(\begin{array}{l}
0 \\
1
\end{array}\right)\right\} \text {, and }\left\{\left(\begin{array}{l}
1 \\
0
\end{array}\right)\right\} \text {. }
$$

If $p$ is bigger than 1 , the only time $x$ is in $\left\{x \mid W_{B}(x)=1\right\}$ is when $l_{0}=l_{1}=\cdots=$ $l_{p-1}$, in which case we are back to the one-cycles.

The crucial step in this argument is the next lemma.

Lemma 9.2. The lattice $\Gamma^{0}=\frac{1}{3} \mathbb{Z} \times \mathbb{Z}$ does not contain any $W_{B}$-cycles of (minimal) period $p>1$.

Proof. A direct computation based on (9.2) above.

Remark 9.3. If $p$ is a multiple of 6 , then there is one $p$-cycle $C$ in $X_{L}$, which is a $W_{B}$-cycle such that $C \cap\left(\frac{1}{3} \mathbb{Z} \times \mathbb{Z}\right) \neq \emptyset$, but no higher cycle is contained in $\frac{1}{3} \mathbb{Z} \times \mathbb{Z}$.

We now relate this to the points $k_{l_{0}, \ldots, l_{p-1}}(\omega)$ in Proposition 8.3 Since for $x \in \mathbb{Z}^{d}$, the four points $S x-l, l \in L$, are distinct, we get a well-defined endomorphism, $\mathcal{R}_{L}: \mathbb{Z}^{2} \rightarrow \mathbb{Z}^{2}$, given by $\mathcal{R}_{L}(S x-l)=x$.

In general, if $C$ is a cycle, set $S(C)=\left\{x \in \mathbb{Z}^{2} \mid\right.$ there is $m \in \mathbb{N}$, s.t. $\left.\mathcal{R}_{L}^{m} x \in C\right\}$. We proved in BrJo99, that

$$
\bigcup_{C} S(C)=\mathbb{Z}^{2}
$$

Moreover it can be checked that the sets $S(C)$ coincide with the points in $\Lambda$ from Proposition 8.3 and Theorem 8.4

The four subsets $S(C) \subset \mathbb{Z}^{2}$ corresponding to the four cycles in (9.3) are simply the four integral quarterplanes which tile $\mathbb{Z}^{2}$. Each quarterplane $S(C)$ has one of the points in the list (9.3) as its vertex:

$$
\begin{aligned}
S\left(\left\{\left(\begin{array}{l}
0 \\
0
\end{array}\right)\right\}\right) & =\left\{\left(\begin{array}{l}
x \\
y
\end{array}\right) \in \mathbb{Z} \mid x \leq 0, y \leq 0\right\}, \\
S\left(\left\{\left(\begin{array}{l}
1 \\
0
\end{array}\right)\right\}\right) & =\left\{\left(\begin{array}{l}
x \\
y
\end{array}\right) \in \mathbb{Z} \mid x \geq 1, y \geq 0\right\}, \\
S\left(\left\{\left(\begin{array}{c}
0 \\
1
\end{array}\right)\right\}\right) & =\left\{\left(\begin{array}{l}
x \\
y
\end{array}\right) \in \mathbb{Z} \mid x \leq 0, y \geq 1\right\}, \\
S\left(\left\{\left(\begin{array}{c}
1 \\
-1
\end{array}\right)\right\}\right) & =\left\{\left(\begin{array}{l}
x \\
y
\end{array}\right) \in \mathbb{Z} \mid x \geq 1, y \leq-1\right\} .
\end{aligned}
$$

Since we already found $\left\{e_{\lambda} \mid \lambda \in \frac{1}{3} \mathbb{Z} \times \mathbb{Z}\right\}$ to be an orthogonal basis in $L^{2}\left(X_{B}\right)$, we conclude that

$$
\sum_{C, W_{B} \text {-cycles }} h_{C}(x)=\operatorname{Proj}_{\mathbb{Z}^{2}}\left(e_{x}\right)<1
$$

unless $e_{x}$ is in the closed span of $\left\{e_{\lambda} \mid \lambda \in \mathbb{Z}^{2}\right\}$. See (6.15).

We conclude by an application of Proposition 8.3 and Theorem 8.4 that $W_{B}$ does not satisfy condition TZ from Definition 5.6. The reader may verify directly the geometric obstruction reflected in condition TZ.

A second consequence of this is that the space $H_{B}(1):=\left\{h \in C\left(X_{L}\right) \mid R_{W_{B}} h=\right.$ $h\}$ has dimension bigger than the number of $W_{B}$-cycles. The only information about the dimension of this eigenspace is that it is finite. This follows from an 
application of the main theorem in [IoMa50]. In particular we conclude that $4<$ $\operatorname{dim}\left(H_{B}(1)\right)<\infty$.

Example 9.4. The next example shares some qualitative features with Example 9.1 above. We outline a system $(B, L, R), \operatorname{det} R=2$ in $\mathbb{R}^{2}$ such that $L^{2}\left(X_{B}\right)$ (with Lebesgue measure) has

$$
\left\{e_{\lambda} \mid \lambda \in \Lambda\right\}=\bigcup_{C, W_{B} \text {-cycles }} S(C)
$$

as an orthogonal basis. Now the $W_{B}$-cycles consist of two one-cycles, a two-cycle, and two four-cycles. For this example we have

$$
\bigcup_{C, W_{B} \text {-cycles }} S(C)=\Lambda=\frac{1}{5} \mathbb{Z} \times \frac{1}{5} \mathbb{Z}
$$

(where $\Lambda$ is the set in Theorem 8.4) and

$$
\sum_{C, W_{B} \text {-cycles }} h_{C}(x)=1, \quad x \in X_{L} .
$$

Specifications $d=2$ :

$$
\begin{gathered}
B=\left\{\left(\begin{array}{l}
0 \\
0
\end{array}\right),\left(\begin{array}{l}
5 \\
0
\end{array}\right)\right\}, \\
L=\left\{\left(\begin{array}{l}
0 \\
0
\end{array}\right),\left(\begin{array}{l}
1 \\
0
\end{array}\right)\right\}, \\
R=\left(\begin{array}{cc}
1 & 1 \\
-1 & 1
\end{array}\right) .
\end{gathered}
$$

In (9.4), $S(C)$ is defined relative to $\Lambda=\frac{1}{5} \mathbb{Z} \times \frac{1}{5} \mathbb{Z}$. With $S=\left(\begin{array}{cc}1 & -1 \\ 1 & 1\end{array}\right)$, it can be checked that $\mathcal{R}_{L}$ may be defined on $\Lambda$. Then

$$
S(C)=\left\{x \in \Lambda \mid \text { there is an } m \text { s.t. } \mathcal{R}_{L}^{m} x \in C\right\}
$$

for any $W_{B}$-cycle $C$.

As in Example 9.1, we check that $\left(R^{-1} B, L\right)$ exponentiates to a Hadamard matrix, in this case $\frac{1}{\sqrt{2}}\left(\begin{array}{cc}1 & 1 \\ 1 & -1\end{array}\right)$, and that the system $\left(\tau_{b}\right)_{b \in B}$ and $\left(\tau_{l}\right)_{l \in L}$ define IFSs $X_{B}$ and $X_{L}$, and $\left\{e_{\lambda} \mid \lambda \in \Lambda\right\}$ is an orthogonal basis for $L^{2}\left(X_{B}\right)$.

The set $X_{L}$ is the twin-dragon from [BrJo99, p. 56, Fig. 2].

Let $l_{0}=\left(\begin{array}{l}0 \\ 0\end{array}\right)$ and $l_{1}=\left(\begin{array}{l}1 \\ 0\end{array}\right)$. Then the two one-cycles are $\left\{\left(l_{0}\right)\right\}$ and $\left\{\left(l_{1}\right)\right\}$, and there is one two-cycle (i.e., with minimal period $=2) C=\left\{\left(l_{0} l_{1}\right),\left(l_{1} l_{0}\right)\right\}$. The two four-cycles are generated by $\left(l_{0} l_{1} l_{1} l_{1}\right)$ and $\left(l_{1} l_{0} l_{0} l_{0}\right)$, respectively. In summary, all these five distinct cycles indeed are $W_{B}$-cycles, and we leave it to the reader to verify that (9.4)-(9.5) are now satisfied. See [BrJo99] for further details.

\section{REFERENCES}

[AST04] A. Aldroubi, Q. Sun, W.S. Tang, Nonuniform average sampling and reconstruction in multiply generated shift-invariant spaces, Constr. Approx. 20 (2004) 173-189. MR2036639 (2005d:42036)

[ALTW04] A. Aldroubi, D. Larson, W.S. Tang, E. Weber, Geometric aspects of frame representations of abelian groups, Trans. Amer. Math. Soc. 356 (2004), 4767-4786. MR2084397 (2005g:42067) 
[Ba00] V. Baladi, Positive Transfer Operators and Decay of Correlations, Advanced Series in Nonlinear Dynamics, vol. 16, World Scientific, River Edge, NJ, 2000. MR1793194 (2001k:37035)

[AtNe04] K.B. Athreya, P.E. Ney, Branching Processes, Dover, Mineola, NY, 2004; reprint of the original edition, Grundlehren Math. Wiss., vol. 196, Springer-Verlag, New YorkHeidelberg, 1972. MR0373040 (51:9242)

[BDP05] S. Bildea, D.E. Dutkay, G. Picioroaga, MRA super-wavelets, New York J. Math. 11 (2005), 1-19. MR2154344 (2006b:42048)

[Bea91] A.F. Beardon, Iteration of Rational Functions: Complex Analytic Dynamical Systems, Graduate Texts in Math., vol. 132, Springer-Verlag, New York, 1991. MR1128089 (92j:30026)

[BJP96] O. Bratteli, P.E.T. Jorgensen, G.L. Price, Endomorphisms of $\mathcal{B}(\mathcal{H})$, Quantization, Nonlinear Partial Differential Equations, and Operator Algebra (Cambridge, MA, 1994) (W. Arveson et al., eds.), Proc. Sympos. Pure Math., vol. 59, American Mathematical Society, Providence, 1996, pp. 93-138. MR1392986 (97h:46107)

[BrJo99] O. Bratteli, P.E.T. Jorgensen, Iterated function systems and permutation representations of the Cuntz algebra, Mem. Amer. Math. Soc. 139 (1999), no. 663. MR.1469149 (99k:46094a)

[BrJo02] O. Bratteli, P.E.T. Jorgensen, Wavelets through a Looking Glass: The World of the Spectrum, Applied and Numerical Harmonic Analysis, Birkhäuser, Boston, 2002. MR.1913212 (2003i:42001)

[Bro65] H. Brolin, Invariant sets under iteration of rational functions, Ark. Mat. 6 (1965), 103-144. MR0194595 (33:2805)

[Che99] X. Chen, Limit theorems for functionals of ergodic Markov chains with general state space, Mem. Amer. Math. Soc. 139 (1999), no. 664. MR1491814 (99k:60170)

[CoRa90] J.-P. Conze, A. Raugi, Fonctions harmoniques pour un opérateur de transition et applications, Bull. Soc. Math. France 118 (1990), 273-310. MR.1078079 (92g:60100)

[Dau92] I. Daubechies, Ten Lectures on Wavelets, CBMS-NSF Regional Conf. Ser. in Appl. Math., vol. 61, SIAM, Philadelphia, 1992. MR1162107 (93e:42045)

[DGS76] M. Denker, C. Grillenberger, K. Sigmund, Ergodic Theory on Compact Spaces, Lecture Notes in Mathematics, vol. 527, Springer-Verlag, Berlin-New York, 1976. MR0457675 $(56: 15879)$

[DuJo03] D.E. Dutkay, P.E.T. Jorgensen, Wavelets on fractals, Rev. Mat. Iberoamericana, to appear, http://arXiv.org/abs/math.CA/0305443.

[DuJo04a] D.E. Dutkay, P.E.T. Jorgensen, Martingales, endomorphisms, and covariant systems of operators in Hilbert space, preprint, 2004, http://arxiv.org/abs/math.CA/0407330 .

[DuJo04b] D.E. Dutkay, P.E.T. Jorgensen, Disintegration of projective measures, Proc. Amer. Math. Soc., to appear, http://arxiv.org/abs/math.CA/0408151.

[DuJo05] D.E. Dutkay, P.E.T. Jorgensen, Hilbert spaces of martingales supporting certain substitution-dynamical systems, Conform. Geom. Dyn. 9 (2005), 24-45. MR2133804 (2006a:37005)

[Fu74] B. Fuglede, Commuting self-adjoint partial differential operators and a group theoretic problem, J. Funct. Anal. 16 (1974), 101-121. MR0470754 (57:10500)

[Gu99] R.F. Gundy, Two remarks concerning wavelets: Cohen's criterion for low-pass filters and Meyer's theorem on linear independence, The Functional and Harmonic Analysis of Wavelets and Frames (San Antonio, TX, 1999) (L.W. Baggett, D.R. Larson, eds.), Contemp. Math., vol. 247, American Mathematical Society, Providence, 1999, pp. 249-258. MR:1738093 (2001b:42051)

[Gu00] R.F. Gundy, Low-pass filters, martingales, and multiresolution analyses, Appl. Comput. Harmon. Anal. 9 (2000), 204-219. MR1777126 (2002k:94013)

[GuKa00] R.F. Gundy, K. Kazarian, Stopping times and local convergence for spline wavelet expansions, SIAM J. Math. Anal. 31 (2000), 561-573. MR.1741041(2001b:42050)

[HMM04] A. Hinkkanen, G.J. Martin, V. Mayer, Local dynamics of uniformly quasiregular mappings, Math. Scand. 95 (2004), 80-100. MR2091483 (2005f:37094)

[Hut81] J.E. Hutchinson, Fractals and self-similarity, Indiana Univ. Math. J. 30 (1981), 713747. MR0625600 (82h:49026) 
[IoMa50] C.T. Ionescu Tulcea, G. Marinescu, Théorie ergodique pour des classes d'opérations non complètement continues, Ann. of Math. (2) 52 (1950), 140-147. MR0037469 $(12: 266 \mathrm{~g})$

[IoPe98] A. Iosevich, S. Pedersen, Spectral and tiling properties of the unit cube, Internat. Math. Res. Notices 1998, 819-828. MR1643694(2000d:52015)

[Jo82] P.E.T. Jorgensen, Spectral theory of finite-volume domains in $\mathbb{R}^{n}$, Adv. Math. 44 (1982), 105-120. MR0658536 (84k:47024)

[Jo05a] P.E.T. Jorgensen, Analysis and Probability: Wavelets, Signals, Fractals, monograph manuscript, book to be published.

[Jo05b] P.E.T. Jorgensen, Measures in wavelet decompositions, Adv. in Appl. Math. 34 (2005), 561-590. MR2123549 (2006b:42053)

[JoPe92] P.E.T. Jorgensen, S. Pedersen, Spectral theory for Borel sets in $\mathbb{R}^{n}$ of finite measure, J. Funct. Anal. 107 (1992), 72-104. MR1165867 (93k:47005)

[JoPe93] P.E.T. Jorgensen, S. Pedersen, Group-theoretic and geometric properties of multivariable Fourier series, Exposition. Math. 11 (1993), 309-329. MR 1240363 (94k:42050)

[JoPe96] P.E.T. Jorgensen, S. Pedersen, Harmonic analysis of fractal measures, Constr. Approx. 12 (1996), 1-30. MR.1389918 (97c:46091)

[JoPe98] P.E.T Jorgensen, S. Pedersen, Dense analytic subspaces in fractal $L^{2}$-spaces, J. Anal. Math. 75 (1998), 185-228. MR1655831 (2000a:46045)

[JoPe99] P.E.T. Jorgensen, S. Pedersen, Spectral pairs in Cartesian coordinates, J. Fourier Anal. Appl. 5 (1999), 285-302. MR1700084 (2002d:42027)

[Ka48] S. Kakutani, On equivalence of infinite product measures, Ann. of Math. (2) 49 (1948), 214-224. MR0023331 (9:340e)

[LaWa02] I. Łaba, Y. Wang. On spectral Cantor measures, J. Funct. Anal. 193 (2002), no. 2, 409-420. MR 1929508 (2003g:28017)

[LRW00] J.C. Lagarias, J.A. Reeds, Y. Wang, Orthonormal bases of exponentials for the n-cube, Duke Math. J. 103 (2000), 25-37. MR1758237(2001h:11104)

[LaSh94] J.C. Lagarias, P.W. Shor, Cube-tilings of $\mathbb{R}^{n}$ and nonlinear codes, Discrete Comput. Geom. 11 (1994), 359-391. MR1273224 (95e:52044)

[LaWa97] J.C. Lagarias, Y. Wang, Integral self-affine tiles in $\mathbb{R}^{n}$, II: Lattice tilings, J. Fourier Anal. Appl. 3 (1997), no. 1, 83-102. MR1428817 (98b:52026)

[LaWa00] J.C. Lagarias, Y. Wang, Orthogonality criteria for compactly supported refinable functions and refinable function vectors, J. Fourier Anal. Appl. 6 (2000), 153-170. MR.1754012 (2002f:42036)

[LMW96] K.-S. Lau, M.-F. Ma, J. Wang, On some sharp regularity estimations of $L^{2}$-scaling functions, SIAM J. Math. Anal. 27 (1996), 835-864. MR1382836 (97d:26001)

[LWC95] K.-S. Lau, J. Wang, C.-H. Chu, Vector-valued Choquet-Deny theorem, renewal equation and self-similar measures, Studia Math. 117 (1995), 1-28. MR1367690 (97m:43001)

[Law91] W.M. Lawton, Necessary and sufficient conditions for constructing orthonormal wavelet bases, J. Math. Phys. 32 (1991), 57-61. MR.1083085 (91m:81100)

[Mane] R. Mañe, On the uniqueness of the maximizing measure for rational maps, Bol. Soc. Brasil. Mat. 14 (1983), 27-43. MR0736567 (85m:58110a)

[MaUr04] R.D. Mauldin, M. Urbański, Graph Directed Markov Systems: Geometry and Dynamics of Limit Sets, Cambridge Tracts in Math., vol. 148, Cambridge University Press, Cambridge, 2003. MR2003772

[NuLu99] R.D. Nussbaum, S.M. Verduyn Lunel, Generalizations of the Perron-Frobenius Theorem for Nonlinear Maps, Mem. Amer. Math. Soc. 138 (1999), no. 659. MR1470912 (99i:58125)

[RW96] S. Richter, R.F. Werner, Ergodicity of quantum cellular automata, J. Stat. Phys. 82 (1996), 963-998. MR.1372433 (97b:82069)

[Rud87] W. Rudin, Real and Complex Analysis, third ed., McGraw-Hill, New York, 1987. MR0924157 (88k:00002)

[Rud91] W. Rudin, Functional Analysis, second ed., Internat. Ser. Pure Appl. Math., McGrawHill, New York, 1991. MR1157815 (92k:46001)

[Rue89] D. Ruelle, The thermodynamic formalism for expanding maps, Comm. Math. Phys. 125 (1989), 239-262. MR.1016871 (91a:58149) 
[SeYa92] J. Seberry, M. Yamada, Hadamard matrices, sequences, and block designs, Contemporary Design Theory: A Collection of Surveys (J.H. Dinitz, D.R. Stinson, eds.), Wiley-Intersci. Ser. Discrete Math. Optim., Wiley, New York, 1992, pp. 431-560. MR 1178508

[So95] B. Solomyak, On the random series $\sum \pm \lambda^{n}$ (an Erdös problem), Ann. of Math. (2) 142 (1995), 611-625. MR.1356783 (97d:11125)

[Str98] R.S. Strichartz, Remarks on "Dense analytic subspaces in fractal $L^{2}$-spaces" by $P$. E. T. Jorgensen and S. Pedersen, J. Anal. Math. 75 (1998), 229-231. MR1655832 (2000a:46046)

[Str00] R.S. Strichartz, Mock Fourier series and transforms associated with certain Cantor measures, J. Anal. Math. 81 (2000), 209-238. MR1785282 (2001i:42009)

[Str05] R.S. Strichartz, Convergence of mock Fourier series, Duke Math. J., to appear.

[Wal75] P. Walters, Ruelle's operator theorem and g-measures, Trans. Amer. Math. Soc. 214 (1975), 375-387. MR0412389 (54:515)

[Wer93] R.F. Werner, Unitary matrices with entries of equal modulus, preprint 1993, Universität Osnabruck.

[YHK97] M. Yamaguti, M. Hata, J. Kigami, Mathematics of Fractals, Transl. Math. Monographs, vol. 167, American Mathematical Society, Providence, 1997. MR1471705 (98j:28006)

[Yo98] K. Yosida, Functional Analysis, Classics in Mathematics, Springer-Verlag, Berlin, 1995; reprint of the sixth edition, Grundlehren Math. Wiss., vol. 123, Springer-Verlag, Berlin-New York, 1980. MR1336382 (96a:46001)

Department of Mathematics, Hill Center-Busch Campus, Rutgers, The State University of New Jersey, 110 Frelinghuysen Rd, Piscataway, New Jersey 08854-8019

E-mail address: ddutkay@math.rutgers.edu

Department of Mathematics, The University of Iowa, 14 Maclean Hall, Iowa City, IOWA 52242-1419

E-mail address: jorgen@math.uiowa.edu 